\title{
1 Metabolic regulation of ILC2 differentiation into 2 ILC1-like cells during Mycobacterium tuberculosis 3 infection
}

4 Dan Corral $^{1,2 \#}$, Alison Charton ${ }^{1}$, Maria Z Krauss $^{3}$, Eve Blanquart ${ }^{4}$, Florence Levillain ${ }^{1}$,

5 Emma Lefrançais ${ }^{1}$, Tamara Sneperger ${ }^{1}$ Jean-Philippe Girard $^{1}$, Gérard Eberl ${ }^{5}$, Yannick

6 Poquet $^{1}$, Jean-Charles Guéry ${ }^{4}$, Rafael J Arguello ${ }^{6}$, Matthew R Hepworth ${ }^{3}$, Olivier

$7 \quad$ Neyrolles $^{1,7}$ and Denis Hudrisier ${ }^{1,7 \#}$

$8{ }^{1}$ Institut de Pharmacologie et Biologie Structurale, IPBS, Université de Toulouse, CNRS, UPS,

9 Toulouse, France

10 2Present address: Metaorganism Immunity Section, Laboratory of Host Immunity and Microbiome,

11 National Institute of Allergy and Infectious Diseases, National Institutes of Health, Bethesda, MD, USA

$12{ }^{3}$ Lydia Becker Institute of Immunology and Inflammation, Division of Infection, Immunity and

13 Respiratory Medicine, School of Biological Sciences, Faculty of Biology, Medicine and Health,

14 Manchester Academic Health Science Centre, University of Manchester, Manchester, M13 9PL, UK

$15{ }^{4}$ Institut Toulousain des Maladies Infectieuses et Inflammatoires (INFINITY), Université de Toulouse,

16 CNRS, UPS, Toulouse, France

175 Institut Pasteur, Microenvironment \& Immunity Unit, 75724 Paris, France ; INSERM U1224, 75724

18 Paris, France

$19{ }^{6}$ Aix Marseille Univ, CNRS, INSERM, CIML, Centre d'Immunologie de Marseille-Luminy, Marseille,

20 France

$21{ }^{7}$ These authors contributed equally: Olivier Neyrolles, Denis Hudrisier

$22 \quad$ \#Co-corresponding authors: Dan Corral (dan.corral@nih.gov); Denis Hudrisier

23 (denis.hudrisier@ipbs.fr) 


\section{Abstract}

25 Tissue-resident innate lymphoid cells (ILCs) regulate tissue homeostasis, protect 26 against pathogens at mucosal surfaces and are key players at the interface of innate 27 and adaptive immunity. How ILCs adapt their phenotype and function to environmental 28 cues within tissues remains to be fully understood. Here, we show that Mycobacterium 29 tuberculosis infection alters the phenotype and function of immature lung ILC2 toward 30 a protective interferon- $\gamma$-producing ILC1-like population. This differentiation is 31 controlled by type 1 cytokines and is associated with a glycolytic program involving the 32 transcription factor HIF1 $\alpha$. Collectively, our data reveal how tissue-resident ILCs adapt to type 1 inflammation toward a pathogen tailored immune response. 


\section{Introduction}

Innate lymphoid cells (ILCs) are a population of tissue-resident cells of lymphoid origin that play a key part in both tissue homeostasis and immunity. ILCs are subdivided into three distinct populations based on their expression of cytokines and specific transcription factors. ILC1 depend on T-bet and produce interferon (IFN)- $\gamma$, ILC2 depend on GATA3 and produce interleukin (IL)-5 and IL-13, and ILC3 depend on ROR $\gamma$ t and produce IL-17A and IL-22 (Meininger et al., 2020; Vivier et al., 2018). Based on these properties, group 1, 2 and 3 ILCs are commonly presented as the innate counterparts of Thelper type 1 (Th1), Th2 and Th17 cells, contributing to type 1, 2 and 3 immune responses, respectively.

The regulome of ILCs evolves progressively during the development of each population to reach a state in which key loci specific to each lineage are acquired (Shih et al., 2016; Vivier et al., 2016). Yet, several elements controlling cytokine expression or loci encoding lineage-determining transcription factors remain broadly accessible in all ILC subsets (Shih et al., 2016). This feature contributes to the remarkable ability of ILCs to dynamically adapt to physiological or pathological alterations in their tissue of residence, and to adopt new phenotypic and functional profiles. Besides the local plasticity among mature ILC subsets (Bal et al., 2020), circulatory and tissue resident ILC precursors in human and mouse contribute to the local differentiation into mature ILCs, an "ILCpoiesis" in situ (Lim and Di Santo, 2019), sustaining the ILC response depending on tissue and inflammation (Ghaedi et al., 2020; Lim et al., 2017; Zeis et al., 2020). While the various populations of tissue-resident ILCs can promptly sense and adapt to environmental changes (Meininger et al., 2020; Ricardo-Gonzalez et al., 2018) the mechanism allowing such responses remains to be fully elucidated.

In both mice and human subjects, Mycobacterium tuberculosis (Mtb) infection induces prolonged proinflammatory responses that are associated with oxidative stress, which favors tissue destruction and triggers a tissue remodeling program. Mtb infection is also associated with metabolic changes in the lungs, involving the utilization of aerobic glycolysis primarily instead of oxidative phosphorylation (OXPHOS) in mitochondria (Warburg effect) (Fernández-García et al., 2020; Shi et al., 2015). At the cytokine level, the lungs at steady-state mostly host resting ILC2, which, together with alveolar macrophages, imprint a type 2-oriented environment to the tissue (Saluzzo et al., 2017; Svedberg et al., 2019). Mtb infection of the lung triggers dramatic changes 
leading to the development of type 1 immunity that is mediated by IFN- $\gamma$ and is associated with protection (O'Garra et al., 2013).

Here, using the murine model of Mtb infection, we explored how lung ILCs respond to chronic pulmonary infection and, in particular, how ILC subsets adapt and respond to type 1 inflammation within tissue. Our work uncovers the local differentiation of lung ILC2 precursors into a protective ILC1-like population through metabolic regulation during Mtb infection.

\section{Results and Discussion}

\section{Local differentiation of ILC2 precursor into ILC1-like cells during Mtb infection}

In order to investigate how a chronic type 1 infection impacts lung ILC subsets, C57BL/6 mice were infected with the Mtb reference strain H37Rv. At steady-state, bona fide lung ILCs were defined as a population that does not express lineage markers (CD3, CD4, CD8, TCR $\beta$, TCRaס, CD49b, CD11b, CD11c, B220, CD19, F4/80, GR-1, TER119, FcعR1a) but highly express CD90.2 and CD45.2 (Figure 1A). ILC2 (GATA3 ${ }^{\text {high }}$ ), which represent the major ILC population in the murine lung, were identified after exclusion of ILC1 $\left(\mathrm{NK} 1.1^{+}\right)$and ILC3 $\left(\mathrm{ROR} \gamma \mathrm{t}^{+}\right)$cells (Figure 1A). At steady state, and in agreement with previously published work (Stehle et al., 2018; Vivier et al., 2018), the lung is dominantly enriched in ILC2 (Figure 1A and 1B). Notably, a small frequency of ILC2 expressed IL-18R $\alpha$ (Figure 1B), a phenotype previously utilized to identify tissue-resident ILC precursor (ILCP) able to differentiate into ILC2 in the context of type 2 inflammatory responses (Ghaedi et al., 2020; Zeis et al., 2020). IL-18R $\alpha^{+}$ILC2 expressed canonical ILC2 markers such as GATA3, ST2, and Arg1 at lower levels than IL-18R $\alpha^{-}$ILC2 (Supplementary Figure 1A-B). At the functional level, these cells produced lower amount of IL-5 compared to IL-18R $\alpha^{-}$ILC2 and did not produce IFN- $\gamma$ like ILC1 (Supplementary Figure 1C). In line with previous studies (Ghaedi et al., 2020; Zeis et al., 2020), we found that IL-18R $\alpha^{+}$ILC2 express high levels of TCF-1, like ILC2 precursors from the bone marrow, confirming their immature profile (Supplementary Figure 1D). Mtb infection had a profound impact on ILC composition and phenotype and was associated with gradual increase in ILC1 and ILC3 (Supplementary Figure 1E). Of particular interest, Mtb infection promoted the 
accumulation of a novel ILC population expressing both IL-18R $\alpha$ and T-bet within the lung (Figure 1A and 1B) and concomitant reduction in IL-18R $\alpha^{-}$ILC2 (Supplementary Figure 1E). Phenotypical analysis revealed that this subset displayed little to no classical ILC2 markers, such as GATA3, ST2, Arg1 and IL-5 (Figure 1C), or ILC3 markers, such as ROR $\gamma$ t (Figure 1A). Like ILC1, this subset expressed T-bet, CD49a, and CD226 (Figure 1D) but did not express NK1.1 (gated on NK1.1 negative ILCs), NKp46 or Eomes (Figure 1A; Supplementary Figure 1E). At the functional level, we found that this population was able to produce IFN- $\gamma$, but not IL-5 or IL-17A (Figure 1E). We therefore named this new subset "ILC1-like cells", based on the similarities (Figure 1D and 1E) and differences (Figure 1A; Supplementary Figure 1F) with NK cells/ILC1. ILC1-like cells became detectable after 21 days of infection and expanded in the following weeks (Figure 1F). Adaptive immune responses are detectable within the lung at 21 days post-Mtb infection (Urdahl et al., 2011), which coincides with the detection of ILC1-like cells. As such we assessed the role of adaptive immunity in the emergence of this ILC subset. ILC1-like cells were detectable in Mtb infected Rag2 ${ }^{-/-}$ mice that lack adaptive immunity, and at a higher level that in infected wild-type mice. Thus, adaptive immunity is not required for the generation of ILC1-like cells following infection (Supplementary Figure 1G).

ILCs have been reported to adapt their profile to environmental cues. Different mechanisms have been described to sustain the local adaptation of ILCs during inflammation such as plasticity of mature ILC subsets (Bal et al., 2020), in situ differentiation of ILC precursor (Ghaedi et al., 2020; Zeis et al., 2020) as well as the migration of ILC from bone marrow (Zeis et al., 2020). Furthermore, ILCs with characteristics of ILC1-like cells have been shown to arise from various ILC subsets through mechanisms of plasticity depending on the tissue and the inflammatory context (Bal et al., 2020; Silver et al., 2016). In the lungs, ILC2 have been described to acquire expression of T-bet, IL-18R $\alpha$ and IFN- $\gamma$ during influenza virus infection (Silver et al., 2016). We hypothesized that ILC1-like cells could differentiate from lung ILC2. To assess if ILC2 display plasticity during Mtb infection, we adoptively transferred total lung ST2+ ILC2, sorted regardless of their IL-18R $\alpha$ expression, into Rag2 ${ }^{-/-} / 12 \mathrm{rg}^{-/-}$mice, which are devoid of T cells, B cells and NK/ILCs, one day prior to Mtb infection (Supplementary Figure 1H and 1I). Before transfer, we confirmed that sorted ILC2 expressed GATA3 but not T-bet or ROR $\gamma$ t (Supplementary Figure 1J) and noticed 
131 that IL-18R $\alpha$ expression was lost during in vitro culture (Supplementary Figure 1J).

132 Following transfer, ILC2 strongly upregulated T-bet in infected, but not in non-infected 133 mice (Supplementary Figure $1 \mathrm{~K}$ and $1 \mathrm{~L}$ ). Furthermore, T-bet $^{\text {high }}$ cells expressed 134 higher level of IL-18R $\alpha$ and Ki67 compared to GATA3 ${ }^{\text {high }}$ cells (Supplementary Figure 135 1M). Given that ILC2 can give rise to ILC1-like cells, we sought to explore which ILC2 136 subset preferentially differentiated into ILC1-like cells. Intriguingly, while IL-18R $\alpha^{+}$ILC2 137 did not acquire ILC1 markers during Mtb infection (Figure 1D) and accumulate into the 138 lungs (Figure 1G), they gained the potential to produce IFN- $\gamma$ and did not produce IL1395 (Figure 1E). Moreover, IL-18R $\alpha^{+}$ILC2, unlike ILC1 and ILC1-like cells, did not 140 respond to ex vivo stimulation with IL-12 and IL-18 (Figure 1H). Thus, we hypothesized 141 that this population could have the potential to differentiate into ILC1-like cells and 142 could thus represent an early stage of ILC1-like. We assessed if IL-18R $\alpha^{+}$ILC2 have 143 the potential to differentiate into ILC1-like during Mtb infection. To this end, we sorted ILC2 subsets from IL-33 treated mice based on their IL-18R $\alpha$ expression and adoptively transferred them into $\mathrm{Rag}^{-{ }^{-I}} / 12 \mathrm{rg}^{-/-}$mice one day before infection with $\mathrm{Mtb}$ (Figure 1I). Interestingly, we found that the transfer of IL-18R $\alpha^{+} \mathrm{ILC} 2$ resulted in higher proportions of ILC in the lungs when compared to the conditions where the same number of IL-18R $\alpha^{-}$ILC2 were transferred (Figure 1J); in addition, T-bet expression among IL-18R $\alpha^{+}$ILC2 was significantly increased in the former case (Figure 1K). Thus, IL-18R $\alpha^{+}$ILC2, rather than IL-18R $\alpha^{-}$ILC2, have the potential to differentiate into ILC1-like cells during Mtb infection.

Altogether, our data show that Mtb infection differentially impacts the composition of ILC subsets within the lung, and especially induces the local differentiation of lung ILC2 precursor into a ILC1-like cell population.

\section{Type 1 inflammatory environment shapes the fate of IL-18R $\alpha^{+}$ILC2}

Next, we aimed to assess how the inflammatory milieu influences the fate of IL-18R $\alpha^{+}$ ILC2. Mtb infection triggers the development of a type 1 immunity (O'Garra et al., 2013). Both IL-12 and IL-18 contribute in the establishment of this type 1 inflammatory environment (Kinjo et al., 2002; O'Garra et al., 2013) in particular by inducing the expression of IFN- $\gamma$ on ILC1, NK cells and Th1 (Chiossone et al., 2018; Weizman et al., 2017). Therefore, we administered to mice IL-12 and IL-18 intranasally for 1 week 
and found that this treatment was sufficient to induce the accumulation of ILC1-like cells in the lungs (Figure 2A-B). Furthermore IL-18R $\alpha^{+}$ILC2 also expanded in these settings (Figure 2B) and lost the expression of TCF-1 (Figure 2C), supporting the idea that these cells may underwent a local differentiation process. Similarly, to Mtb infection, IL-18R $\alpha^{+}$ILC2, and IL-18R $\alpha^{-}$ILC2, lost their ability to produce IL-5 following cytokine injection and acquired the ability to produce IFN- $\gamma$ (Figure 2D), but not after ex vivo stimulation with IL-12 and IL-18 (Figure 2E). Moreover, we crossed IL-5 tdTomato (Red5) mice with ROSA26-YFP mice to enable fate-mapping mature ILC2 (Nussbaum et al., 2013) and that the majority of ILC1-like cell do not derive from IL$18 \mathrm{R} \alpha-$ ILC2, the only ILC population expressing IL-5 at steady-state and during type 1 inflammation (Supplementary Figure 2A-B), reinforcing our previous observation (Figure 1J). Overall, based on the expression of several markers (GATA3, Arg1, Tbet, IL-18R $\alpha$, CD49a, CD226 and Ki67), we found close similarities in both IL-18R $\alpha^{+}$ ILC2 and ILC1-like cells generated upon either IL-12/IL-18 treatment or during Mtb infection (Supplementary Figure 2B). Thus, the generation of ILC1-like cells observed during Mtb infection can be closely recapitulated with the simple administration of IL-12 and IL-18.

To further demonstrate the ILC2 origin of ILC1-like cells during type 1 inflammation, we studied the effect of IL-33, a well-known inducer of both mature and immature ILC2 (Moro et al., 2010; Neill et al., 2010; Price et al., 2010), on ILC1-like differentiation. IL33 alone did not induce the differentiation of ILC1-like cells, although it did induce a high expansion of mature and immature ILC2 (Figure 2F-I). In association with IL-12 and IL-18, IL-33 was able to enhance ILC1-like differentiation (Figure 2I and Supplementary Figure 2C). Intriguingly, while IL-18R $\alpha^{+}$ILC2 expressed ILC2 markers (ST2, Arg1 and IL-5) in IL-33-treated mice (Figure 2J-K), and ILC1-like markers (CD49a, IFN- $\gamma$ ) in IL-12/IL-18-treated animals (Figure 2J-K), the combination of IL-12/IL-18 with IL-33 led to a mixed ILC1/ILC2 phenotype with the capacity to produce both IL-5 and IFN- $\gamma$. Because ST2 is expressed by various cell types, including ILC2, we also tested Neuromedin U (NMU), whose receptor is solely present in bone marrow ILC2P and in lung ILC2 (Cardoso et al., 2017; Klose et al., 2014; Wallrapp et al., 2017). Similar to IL-33, NMU potentiated the differentiation of ILC1-like cells induced by IL-12 and IL-18 (Figure 2L). Altogether, these results demonstrate that lung IL-18R $\alpha^{+}$ILC2 exhibit a highly adaptable phenotype, dependent on the 
inflammatory environment. While they strengthen the ILC2 response in a type 2 environment (i.e., after administration of IL-33), these cells rather differentiate into IFN$\gamma$-producing ILC1-like cells in a type 1 environment (i.e., after administration of IL-12 and IL-18). This result supports the recent notion that local ILC precursors may undergo "ILCpoiesis" (Ghaedi et al., 2020; Zeis et al., 2020), as demonstrated in human (Lim et al., 2017; Lim and Di Santo, 2019). Although we cannot exclude local plasticity of other ILC subsets, or ILCP recruitment from the bone marrow, our results strongly suggest the local differentiation of lung ILC2P into ILC1-like cells during type 1 inflammation.

\section{ILC1-like cell differentiation is associated with a metabolic reprogramming toward glycolysis.}

Recent RNA-sequencing analyses of intestinal ILCs revealed that each subset display unique metabolic profiles (Gury-BenAri et al., 2016). While the need in amino acid metabolism for lung ILC2 functions relies on Arg1 (Monticelli et al., 2016), the glycolytic pathway necessary for ILC3 functions depends on mTOR and HIF1 $\alpha$ (Di Luccia et al., 2019). However, little is known about metabolic adaptation of ILCs to their environment during infection (Joseph et al., 2018). Fate decisions of immune cells such as those underlying differentiation of Treg/Th17 or Treg/Th1 have been tightly associated with metabolic reprogramming (Clever et al., 2016; Dang et al., 2011; Shi et al., 2011). Given that IL-18R $\alpha^{+}$ILC2 present the ability to differentiate into ILC1-like cells in a type 1 inflammatory context, we investigated the metabolic pathways engaged during ILC1-like cells differentiation. To gain insight in ILC metabolism, we took advantage of the recently described SCENITH method (Argüello et al., 2020), which allows to determine global metabolic dependencies and capacities at the single cell level. SCENITH uses protein synthesis levels as a readout and is particularly appropriate to analyze the metabolism of rare cells, such as ILCs. ILC1-like cells were compared to control cells known to rely on a glycolytic metabolism (e.g., NK cells) and to ILC2 in lungs. In agreement with the inhibitory effect of type 1 inflammation on IL18R $\alpha^{-}$ILC2 (Figure 1E; Supplementary Figure 1F), administration of IL-12 and IL-18 downregulated ILC2 global level of translation, as assessed via detection of puromycin incorporation (Figure 3A-C). Conversely, following cytokine injection, the level of translation was increased in NK cells, IL-18R $\alpha^{+}$ILC2 and ILC1-like cells with the latter 
cells displaying the highest rate (Figure 3A-C). Notably, a similar pattern was observed during Mtb infection (Supplementary Figure 3A). The analysis of protein synthesis in the presence of inhibitors targeting different metabolic pathways, namely 2-DG for glycolysis and oligomycin for OXPHOS, allowed us to assess the mitochondrial dependence and glycolytic capacity of the cells (Figure 3D). We found that, in all ILC subsets tested, type 1 inflammation led to a global decrease in their mitochondrial dependence, together with an increase in their glycolytic capacity, that is a canonical feature of the Warburg effect (Heiden et al., 2009) (Figure 3E and F). Thus, while a metabolic reprogramming towards glycolysis is significantly induced in IL-18R $\alpha^{-}$ILC2, IL-18R $\alpha^{+}$ILC2 and characterized ILC1-like cells upon type 1 inflammation, this program was also associated with a global inhibition of IL-18R $\alpha^{-}$ILC2 compared to the other ILC subsets tested. Arg1 has been previously identified as a critical component of the metabolic programming of lung ILC2, with its inhibition or genetic inactivation resulting in reduced aerobic glycolysis (Monticelli et al., 2016). In agreement with previous studies (Bando et al., 2013; Monticelli et al., 2016; Schneider et al., 2019), we found that Arg1 was highly expressed in both IL-18R $\alpha^{-}$and IL-18R $\alpha^{+}$ILC2 (Figure 3G). However, after treatment with IL-12 and IL-18, the expression of Arg1 decreased in IL-18R $\alpha^{+}$ILC2 to similar level as ILC1-like cells, supporting the idea that Arg1 is not implicated in the metabolic regulation of ILC1-like cell differentiation. Previous work proposed that the Warburg effect, a metabolic pathway that is engaged during Mtb infection (Fernández-García et al., 2020; Shi et al., 2015), relies on the transcription factor hypoxia-inducible factor-1 a (HIF1a) (Palazon et al., 2014). Of interest, in a model of von Hippel-Lindau (VHL) deficiency, where HIF1 $\alpha$ is overexpressed, it was previously shown that ILC2 development was repressed through glycolysis induction (Li et al., 2018). These observations prompted us to analyze HIF1 $\alpha$ expression in our model. Type 1 inflammation driven by IL-12/IL-18 treatment led to the induction of HIF1 $\alpha$ in both IL-18R $\alpha^{-}$and IL-18R $\alpha^{+}$ILC2, and in ILC1-like cells (Figure 3H). Given that IL-18R $\alpha^{-}$and IL-18R $\alpha^{+}$ILC2 share the same metabolic dependence but differ in their activation state during type 1 inflammation, we analyzed the impact of HIF $1 \alpha$ expression on ILC2. We performed an in vitro assay using sorted ILC2 cultured in the presence of DMOG, which stabilizes the HIF1 $\alpha$ protein (Palazon et al., 2014) (Figure 3I). DMOG-treatment had a significant inhibitory impact on expression of ILC2 markers including GATA3, ST2 and IL-5 (Figure 3J-L). Accordingly, analysis of the global 
metabolic profile of ILC2 revealed that DMOG-treated ILC2 harbored a glycolytic profile (Figure 3M), while untreated ILC2 preferentially used mitochondrial respiration (Figure 3N). Most notably, DMOG treatment alone was sufficient to upregulate genes typically associated with an ILC1 phenotype, such as Tbx21, Ifng and II18r1 (Figure 30). Altogether, these results suggest that HIF1 $\alpha$ in lung ILC2 promote a metabolic reprogramming toward glycolysis, while favoring the acquisition of an ILC1-like profile.

Next, we sought to determine the role of glycolysis in the differentiation of ILC1like cells during Mtb infection. First, we observed that HIF1 $\alpha$ is expressed in all ILC subsets, with the highest levels in ILC3 and ILC1-like cells (Supplementary Figure 3B). Inhibition of glycolysis during ex vivo stimulation of total lung ILCs decreased the proportion of IFN- $\gamma^{+}$ILCs (Supplementary Figure 3C), showing that IFN- $\gamma$ production is glycolysis-dependent. To assess the impact of glycolysis induction in vivo, we first treated mice with 2-deoxyglucose (2-DG), a glycolysis inhibitor, during Mtb infection. 2-DG administration markedly decreased the number of ILC1-like cells as well as their ability to produce IFN- $\gamma$ (Supplementary Figure 3D, E). Next, since glucose is consumed in the lungs of Mtb-infected mice (Fernández-García et al., 2020), we investigated if the modulation of glucose availability in the lung environment could modulate the differentiation of ILC1-like cells. Glucose supplementation in the animals' drinking water enhanced the differentiation of ILC1-like cells (Supplementary Figure 3F) and increased the percentage of IFN- $\gamma^{+}$ILC1-like cells (Supplementary Figure 3G). Thus, these results strongly suggest that glycolysis is required to support ILC1like cells differentiation and function during Mtb infection.

\section{ILC1-like cells confer protection against $M$ tb}

Next, we investigated whether BCG, the only available vaccine for TB, might impact the population of lung-resident ILCs when delivered intranasally, a route providing a better protection than the conventional subcutaneous route (Perdomo et al., 2016), prior to Mtb infection (Figure 4A). As expected, mucosal BCG vaccination induced protection upon Mtb challenge (Figure 4B). In vaccinated mice, protection correlated with an increase in T-bet expression in ILCs (Figure 4C). More importantly, although BCG vaccination had no impact on other ILC subsets (Figure 4D), higher numbers of 
ILC1-like cells were virtually absent from non-vaccinated mice (Figure 4D, E) but wellinduced in vaccinated animals (Figure 4D, E). Overall, BCG vaccination promotes ILC1-like cells in early stages of infection, which could contribute to protection against Mtb.

Finally, to assess the contribution of ILC1-like cells to protection against Mtb, we took advantage of the cytokine-induced ILC1-like cell model (Figure 4F) to generate enough ILC1-like cells for adoptive transfer. ILC1-like cells were sorted from the lungs of mice treated with IL-12, IL-18 and IL-33; these cells expressed T-bet, but not GATA3 or ROR $\gamma$ t (Figure 4G). Remarkably, the transfer of as few as 10,000 ILC1-like cells resulted in a statistically significant reduction in bacterial load after Mtb challenge, demonstrating the protective capacity of ILC1-like cells against the tuberculosis bacillus (Figure 4H). Recently, ILC3 were reported to mediate protection against Mtb through induction of lung ectopic lymphoid follicles (Ardain et al., 2019). Although our results confirm the expansion and activation of ILC3 during Mtb infection (Figure 1E ; Supplementary Figure 1F), we report the expansion of an ILC1-like cell population, which can contribute to protection against Mtb infection. Differences between the two studies may be due to the Mtb strains used, namely HN878 (Ardain et al., 2019) vs. H37Rv in our study, the different proportions of the various ILC subsets found in the lungs, or both. In particular, infection with the hypervirulent strain HN878 is known to induce a different inflammatory pattern (e.g. with strong production of IL-1 $\beta$ and type 1 IFN) and protective immune mechanisms (e.g. IL-17 and IL-22 production) compared to H37Rv (Gopal et al., 2014; Manca et al., 2001). Thus, depending on the strain and the associated inflammation, ILC subsets might be highly impacted in their regulation and function during infection.

Thus, we propose a model in which the local differentiation of lung ILC2 precursor into ILC1-like cells is regulated by both inflammatory and metabolic environment induced by Mtb infection (Supplementary Figure 4). Our observation that BCG vaccination favors the early generation of ILC1-like cells and that ILC1-like cell are endowed with a protective potential during $M t b$ infection lead to future studies aiming at elucidating the role played by ILC1-like cells in protection. On a broader perspective, targeting ILC1-like cells using dedicated strategies may help develop novel approaches to combat tuberculosis and other inflammatory diseases. 


\section{References}

325 Ardain, A., Domingo-Gonzalez, R., Das, S., Kazer, S.W., Howard, N.C., Singh, A., Ahmed, M., Nhamoyebonde, S., Rangel-Moreno, J., Ogongo, P., Lu, L., Ramsuran, D., de la Luz Garcia-Hernandez, M., K. Ulland, T., Darby, M., Park, E., Karim, F., Melocchi, L., Madansein, R., Dullabh, K.J., Dunlap, M., Marin-Agudelo, N., Ebihara, T., Ndung'u, T., Kaushal, D., Pym, A.S., Kolls, J.K., Steyn, A., Zúñiga, J., Horsnell, W., Yokoyama, W.M., Shalek, A.K., Kløverpris, H.N., Colonna, M., Leslie, A., Khader, S.A., 2019. Group 3 innate lymphoid cells mediate early protective immunity against tuberculosis. Nature 570, 528-532. https://doi.org/10.1038/s41586-019-1276-2

Argüello, R.J., Combes, A.J., Char, R., Gigan, J.-P., Baaziz, A.I., Bousiquot, E.,

Based Method to Functionally Profile Energy Metabolism with Single-Cell Resolution.

Cell Metab. 32, 1063-1075.e7. https://doi.org/10.1016/j.cmet.2020.11.007

Bal, S.M., Golebski, K., Spits, H., 2020. Plasticity of innate lymphoid cell subsets. Nat.

Bando, J.K., Nussbaum, J.C., Liang, H.-E., Locksley, R.M., 2013. Type 2 innate lymphoid cells constitutively express arginase-I in the naïve and inflamed lung. J. Leukoc. Biol. 94, 877-884. https://doi.org/10.1189/jlb.0213084

Cardoso, V., Chesné, J., Ribeiro, H., García-Cassani, B., Carvalho, T., Bouchery, T., regulation of type 2 innate lymphoid cells via neuromedin U. Nature 549, 277-281. https://doi.org/10.1038/nature23469

Chiossone, L., Dumas, P.-Y., Vienne, M., Vivier, E., 2018. Natural killer cells and other innate lymphoid cells in cancer. Nat. Rev. Immunol. 18, 671-688. https://doi.org/10.1038/s41577-018-0061-z 
integrate glycolysis and mitochondrial production of reactive oxygen species to fulfill activation demands. J. Exp.

Med.

216,

2231-2241.

https://doi.org/10.1084/jem.20180549

Fernández-García, M., Rey-Stolle, F., Boccard, J., Reddy, V.P., García, A., Cumming, B.M., Steyn, A.J.C., Rudaz, S., Barbas, C., 2020. Comprehensive Examination of the Mouse Lung Metabolome Following Mycobacterium tuberculosis Infection Using a Multiplatform Mass Spectrometry Approach. J. Proteome Res. 19, 2053-2070. https://doi.org/10.1021/acs.jproteome.9b00868

Ghaedi, M., Shen, Z.Y., Orangi, M., Martinez-Gonzalez, I., Wei, L., Lu, X., Das, A., Heravi-Moussavi, A., Marra, M.A., Bhandoola, A., Takei, F., 2020. Single-cell analysis of RORa tracer mouse lung reveals ILC progenitors and effector ILC2 subsets. J. Exp. Med. 217. https://doi.org/10.1084/jem.20182293

Gopal, R., Monin, L., Slight, S., Uche, U., Blanchard, E., Fallert Junecko, B.A., RamosPayan, R., Stallings, C.L., Reinhart, T.A., Kolls, J.K., Kaushal, D., Nagarajan, U., Rangel-Moreno, J., Khader, S.A., 2014. Unexpected role for IL-17 in protective immunity against hypervirulent Mycobacterium tuberculosis HN878 infection. PLoS Pathog. 10, e1004099. https://doi.org/10.1371/journal.ppat.1004099

Gury-BenAri, M., Thaiss, C.A., Serafini, N., Winter, D.R., Giladi, A., Lara-Astiaso, D., Levy, M., Salame, T.M., Weiner, A., David, E., Shapiro, H., Dori-Bachash, M., PevsnerFischer, M., Lorenzo-Vivas, E., Keren-Shaul, H., Paul, F., Harmelin, A., Eberl, G., Itzkovitz, S., Tanay, A., Di Santo, J.P., Elinav, E., Amit, I., 2016. The Spectrum and Regulatory Landscape of Intestinal Innate Lymphoid Cells Are Shaped by the Microbiome. Cell 166, 1231-1246.e13. https://doi.org/10.1016/j.cell.2016.07.043

Heiden, M.G.V., Cantley, L.C., Thompson, C.B., 2009. Understanding the Warburg Effect: The Metabolic Requirements of Cell Proliferation. Science 324, 1029-1033. https://doi.org/10.1126/science.1160809 and adaptive lymphocyte effector responses. Immunol. Rev. 286, 137-147. 
https://doi.org/10.1111/imr.12703

Kinjo, Y., Kawakami, K., Uezu, K., Yara, S., Miyagi, K., Koguchi, Y., Hoshino, T., Okamoto, M., Kawase, Y., Yokota, K., Yoshino, K., Takeda, K., Akira, S., Saito, A., 2002. Contribution of IL-18 to Th1 response and host defense against infection by Mycobacterium tuberculosis: a comparative study with IL-12p40. J. Immunol. Baltim. Md 1950 169, 323-329. https://doi.org/10.4049/jimmunol.169.1.323

Klose, C.S.N., Flach, M., Möhle, L., Rogell, L., Hoyler, T., Ebert, K., Fabiunke, C., Pfeifer, D., Sexl, V., Fonseca-Pereira, D., Domingues, R.G., Veiga-Fernandes, H., Arnold, S.J., Busslinger, M., Dunay, I.R., Tanriver, Y., Diefenbach, A., 2014. Differentiation of Type $1 \mathrm{lLCs}$ from a Common Progenitor to All Helper-like Innate Lymphoid Cell Lineages. Cell 157, 340-356. https://doi.org/10.1016/j.cell.2014.03.030

Li, Q., Li, D., Zhang, X., Wan, Q., Zhang, W., Zheng, M., Zou, L., Elly, C., Lee, J.H., Liu, Y.-C., 2018. E3 Ligase VHL Promotes Group 2 Innate Lymphoid Cell Maturation and Function via Glycolysis Inhibition and Induction of Interleukin-33 Receptor. Immunity 48, 258-270.e5. https://doi.org/10.1016/j.immuni.2017.12.013

Lim, A.I., Di Santo, J.P., 2019. ILC-poiesis: Ensuring tissue ILC differentiation at the right place and time. Eur. J. Immunol. 49, 11-18. https://doi.org/10.1002/eji.201747294

Lim, A.I., Li, Y., Lopez-Lastra, S., Stadhouders, R., Paul, F., Casrouge, A., Serafini, N., Puel, A., Bustamante, J., Surace, L., Masse-Ranson, G., David, E., StrickMarchand, H., Bourhis, L.L., Cocchi, R., Topazio, D., Graziano, P., Muscarella, L.A., Rogge, L., Norel, X., Sallenave, J.-M., Allez, M., Graf, T., Hendriks, R.W., Casanova, J.-L., Amit, I., Yssel, H., Santo, J.P.D., 2017. Systemic Human ILC Precursors Provide a Substrate for Tissue ILC Differentiation. Cell 168, 1086-1100.e10. https://doi.org/10.1016/j.cell.2017.02.021

Manca, C., Tsenova, L., Bergtold, A., Freeman, S., Tovey, M., Musser, J.M., Barry, C.E., Freedman, V.H., Kaplan, G., 2001. Virulence of a Mycobacterium tuberculosis clinical isolate in mice is determined by failure to induce Th1 type immunity and is associated with induction of IFN- $\alpha / \beta$. Proc. Natl. Acad. Sci. 98, 5752-5757. 
https://doi.org/10.1073/pnas.091096998

Meininger, I., Carrasco, A., Rao, A., Soini, T., Kokkinou, E., Mjösberg, J., 2020. TissueSpecific Features of Innate Lymphoid Cells. Trends Immunol. 41, 902-917. https://doi.org/10.1016/j.it.2020.08.009

Monticelli, L.A., Buck, M.D., Flamar, A.-L., Saenz, S.A., Tait Wojno, E.D., Yudanin, N.A., Osborne, L.C., Hepworth, M.R., Tran, S.V., Rodewald, H.-R., Shah, H., Cross, J.R., Diamond, J.M., Cantu, E., Christie, J.D., Pearce, E.L., Artis, D., 2016. Arginase 1 is an innate lymphoid-cell-intrinsic metabolic checkpoint controlling type 2 inflammation. Nat. Immunol. 17, 656-665. https://doi.org/10.1038/ni.3421

Moro, K., Yamada, T., Tanabe, M., Takeuchi, T., Ikawa, T., Kawamoto, H., Furusawa, J., Ohtani, M., Fujii, H., Koyasu, S., 2010. Innate production of T H 2 cytokines by adipose tissue-associated c-Kit + Sca-1 + lymphoid cells. Nature 463, 540-544. https://doi.org/10.1038/nature08636

Neill, D.R., Wong, S.H., Bellosi, A., Flynn, R.J., Daly, M., Langford, T.K.A., Bucks, C., Kane, C.M., Fallon, P.G., Pannell, R., Jolin, H.E., McKenzie, A.N.J., 2010. Nuocytes represent a new innate effector leukocyte that mediates type-2 immunity. Nature 464, 1367-1370. https://doi.org/10.1038/nature08900

Nussbaum, J.C., Van Dyken, S.J., von Moltke, J., Cheng, L.E., Mohapatra, A., Molofsky, A.B., Thornton, E.E., Krummel, M.F., Chawla, A., Liang, H.-E., Locksley, R.M., 2013. Type 2 innate lymphoid cells control eosinophil homeostasis. Nature 502 , 245-248. https://doi.org/10.1038/nature12526

O'Garra, A., Redford, P.S., McNab, F.W., Bloom, C.I., Wilkinson, R.J., Berry, M.P.R., 2013. The Immune Response in Tuberculosis. Annu. Rev. Immunol. 31, 475-527. https://doi.org/10.1146/annurev-immunol-032712-095939

Palazon, A., Goldrath, A.W., Nizet, V., Johnson, R.S., 2014. HIF Transcription Factors, Inflammation, and Immunity. Immunity 41, 518-528. https://doi.org/10.1016/j.immuni.2014.09.008 
459 Perdomo, C., Zedler, U., Kühl, A.A., Lozza, L., Saikali, P., Sander, L.E., Vogelzang,

460

461

462

463

464

465

466

467

468

469

470

471

472

473

474

475

476

477

478

479

480

481

482

483

484

485

486

487

488

489

490

491

492

A., Kaufmann, S.H.E., Kupz, A., 2016. Mucosal BCG Vaccination Induces Protective Lung-Resident Memory $T$ Cell Populations against Tuberculosis. mBio 7. https://doi.org/10.1128/mBio.01686-16

Price, A.E., Liang, H.-E., Sullivan, B.M., Reinhardt, R.L., Eisley, C.J., Erle, D.J., Locksley, R.M., 2010. Systemically dispersed innate IL-13-expressing cells in type 2 immunity. Proc. Natl. Acad. Sci. 107, 11489-11494. https://doi.org/10.1073/pnas.1003988107

Ricardo-Gonzalez, R.R., Van Dyken, S.J., Schneider, C., Lee, J., Nussbaum, J.C., Liang, H.-E., Vaka, D., Eckalbar, W.L., Molofsky, A.B., Erle, D.J., Locksley, R.M., 2018. Tissue signals imprint ILC2 identity with anticipatory function. Nat. Immunol. 19, 10931099. https://doi.org/10.1038/s41590-018-0201-4

Saluzzo, S., Gorki, A.-D., Rana, B.M.J., Martins, R., Scanlon, S., Starkl, P., Lakovits, K., Hladik, A., Korosec, A., Sharif, O., Warszawska, J.M., Jolin, H., Mesteri, I., McKenzie, A.N.J., Knapp, S., 2017. First-Breath-Induced Type 2 Pathways Shape the Lung Immune Environment. Cell Rep. 18, 1893-1905. https://doi.org/10.1016/j.celrep.2017.01.071

Schneider, C., Lee, J., Koga, S., Ricardo-Gonzalez, R.R., Nussbaum, J.C., Smith, L.K., Villeda, S.A., Liang, H.-E., Locksley, R.M., 2019. Tissue-Resident Group 2 Innate Lymphoid Cells Differentiate by Layered Ontogeny and In Situ Perinatal Priming. Immunity 50, 1425-1438.e5. https://doi.org/10.1016/j.immuni.2019.04.019

Shi, L., Salamon, H., Eugenin, E.A., Pine, R., Cooper, A., Gennaro, M.L., 2015. Infection with Mycobacterium tuberculosis induces the Warburg effect in mouse lungs. Sci. Rep. 5, 1-13. https://doi.org/10.1038/srep18176

Shih, H.-Y., Sciumè, G., Mikami, Y., Guo, L., Sun, H.-W., Brooks, S.R., Urban, J.F., Davis, F.P., Kanno, Y., O'Shea, J.J., 2016. Developmental Acquisition of Regulomes Underlies Innate Lymphoid Cell Functionality. Cell 165, 1120-1133. https://doi.org/10.1016/j.cell.2016.04.029 
493 Silver, J.S., Kearley, J., Copenhaver, A.M., Sanden, C., Mori, M., Yu, L., Pritchard, 494 G.H., Berlin, A.A., Hunter, C.A., Bowler, R., Erjefalt, J.S., Kolbeck, R., Humbles, A.A., 495 2016. Inflammatory triggers associated with exacerbations of COPD orchestrate plasticity of group 2 innate lymphoid cells in the lungs. Nat. Immunol. 17, 626-635. https://doi.org/10.1038/ni.3443

498

499

Svedberg, F.R., Brown, S.L., Krauss, M.Z., Campbell, L., Sharpe, C., Clausen, M., 500 Howell, G.J., Clark, H., Madsen, J., Evans, C.M., Sutherland, T.E., Ivens, A.C.,

501

502

503

504

505

506

507

508

509

510

511

512

513

514

515

516

517

518

519

520

521

522

523

524

525

526

Thornton, D.J., Grencis, R.K., Hussell, T., Cunoosamy, D.M., Cook, P.C., MacDonald, A.S., 2019. The lung environment controls alveolar macrophage metabolism and responsiveness in type 2 inflammation. Nat. Immunol. 20, 571-580. https://doi.org/10.1038/s41590-019-0352-y

Troegeler, A., Mercier, I., Cougoule, C., Pietretti, D., Colom, A., Duval, C., Vu Manh, T.-P., Capilla, F., Poincloux, R., Pingris, K., Nigou, J., Rademann, J., Dalod, M., Verreck, F.A.W., Al Saati, T., Lugo-Villarino, G., Lepenies, B., Hudrisier, D., Neyrolles, O., 2017. C-type lectin receptor DCIR modulates immunity to tuberculosis by sustaining type I interferon signaling in dendritic cells. Proc. Natl. Acad. Sci. U. S. A. 114, E540-E549. https://doi.org/10.1073/pnas.1613254114

Urdahl, K., Shafiani, S., Ernst, J., 2011. Initiation and regulation of T-cell responses in tuberculosis. Mucosal Immunol. 4, 288-293. https://doi.org/10.1038/mi.2011.10

Vivier, E., Artis, D., Colonna, M., Diefenbach, A., Santo, J.P.D., Eberl, G., Koyasu, S., Locksley, R.M., McKenzie, A.N.J., Mebius, R.E., Powrie, F., Spits, H., 2018. Innate Lymphoid Cells: 10 Years On. Cell 174, 1054-1066. https://doi.org/10.1016/j.cell.2018.07.017

Vivier, E., van de Pavert, S.A., Cooper, M.D., Belz, G.T., 2016. The evolution of innate lymphoid cells. Nat. Immunol. 17, 790-794. https://doi.org/10.1038/ni.3459

Wallrapp, A., Riesenfeld, S.J., Burkett, P.R., Abdulnour, R.-E.E., Nyman, J., Dionne, D., Hofree, M., Cuoco, M.S., Rodman, C., Farouq, D., Haas, B.J., Tickle, T.L., Trombetta, J.J., Baral, P., Klose, C.S.N., Mahlakõiv, T., Artis, D., Rozenblatt-Rosen, 
527 O., Chiu, I.M., Levy, B.D., Kowalczyk, M.S., Regev, A., Kuchroo, V.K., 2017. The 528 neuropeptide NMU amplifies ILC2-driven allergic lung inflammation. Nature 549, 351529 356. https://doi.org/10.1038/nature24029

530

531 Weizman, O.-E., Adams, N.M., Schuster, I.S., Krishna, C., Pritykin, Y., Lau, C., Degli532 Esposti, M.A., Leslie, C.S., Sun, J.C., O’Sullivan, T.E., 2017. ILC1 Confer Early Host 533 Protection at Initial Sites of Viral Infection. Cell 171, 795-808.e12. 534 https://doi.org/10.1016/j.cell.2017.09.052

535

536 Zeis, P., Lian, M., Fan, X., Herman, J.S., Hernandez, D.C., Gentek, R., Elias, S., 537 Symowski, C., Knöpper, K., Peltokangas, N., Friedrich, C., Doucet-Ladeveze, R., 538 Kabat, A.M., Locksley, R.M., Voehringer, D., Bajenoff, M., Rudensky, A.Y., 539 Romagnani, C., Grün, D., Gasteiger, G., 2020. In Situ Maturation and Tissue 540 Adaptation of Type 2 Innate Lymphoid Cell Progenitors. Immunity 53, 775-792.e9. 541 https://doi.org/10.1016/j.immuni.2020.09.002 


\section{Materials \& Methods}

544

545

546

547

548

549

550

551

552

553

554

555

556

557

558

559

560

561

562

563

564

565

566

567

568

569

570

571

572

573

574

\section{Mice}

Six-to-eight-week-old female C57BL/6 mice were purchased from Charles River Laboratories France (Saint Germain Nuelles, France). Rag2-/- (B6.129-Rag2tm1Fwa), Red5 mice (B6(C)-II5tm1.1(icre)Lky/J)n, Rag2 ${ }^{-1-} \gamma_{c}{ }^{-/-} \quad$ (C;129S4-Rag2tm1.1Flv II2rgtm1.1Flv/J) on a C57BL/6 J were bred in our animal facility. ROSA26-YFP mice

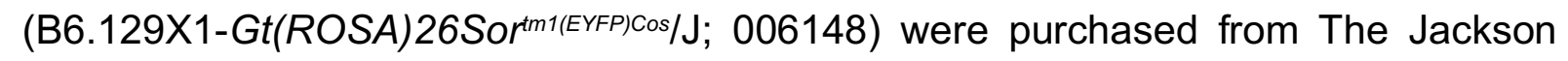
Laboratory through Charles Rivers Laboratory France. All mice were maintained in specific-pathogen-free animal facility at IPBS and all experiments were conducted in strict accordance with French laws and regulations in compliance with the European Community council directive 68/609/EEC guidelines and its implementation in France under procedures approved by the French Ministry of Research and the FRBT (C2EA01) animal care committee (APAFIS \#1269, \#3873, \#10546, \#16529 and \#17384).

\section{Mtb culture, immunization \& mouse infections}

The laboratory strain of $\mathrm{Mtb}, \mathrm{H} 37 \mathrm{Rv}$, was grown at $37^{\circ} \mathrm{C}$ in Middlebrook $7 \mathrm{H} 9$ medium (Difco) supplemented with 10\% albumin-dextrose-catalase (ADC, Difco) and 0.05\% Tyloxapol (Sigma), or on Middlebrook 7H11 agar medium (Difco) supplemented with $10 \%$ oleic acid-albumin-dextrose-catalase (OADC, Difco). Six- to eight-week-old mice were anesthetized with a cocktail of ketamine $(60 \mathrm{mg} / \mathrm{kg}$, Merial) and xylasine (10 $\mathrm{mg} / \mathrm{kg}$, Bayer) and infected intranasally (i.n.) with 1000 CFUs of mycobacteria in $25 \mu \mathrm{L}$ of PBS containing $0.01 \%$ Tween 80 . For immunization, C57BL/B6 mice were immunized i.n. with $5.10^{5}$ CFU of BCG (Danish), and were challenged 60 days postvaccination with H37Rv as previously described. All experiments using Mtb were performed in appropriate biosafety level 3 (BSL3) laboratory and animal facility.

\section{In vivo treatments}

2-DG (1 $\mathrm{g} / \mathrm{kg}$, Sigma) was injected every other day starting from the day of infection and until completion of the experiment. For glucose supplementation, mice were 
575 treated with drinking water containing $30 \%(\mathrm{w} / \mathrm{v})$ glucose (started 1 week before

576 infection until sacrifice).

577

578 Adoptive transfer experiments

579

580 For the adoptive transfer of total lung ILC2, in vitro cultured of ILC2 were harvested 581 after 7 days of culture and $5 \times 10^{5}$ to $2 \times 10^{6}$ cells were transferred i.v. in mice anesthetized with isoflurane one day before Mtb infection in $\mathrm{Rag}^{-1-} \gamma_{c}{ }^{-/-}$. For the adoptive transfer of the IL-18R $\alpha^{-}$or IL-18R $\alpha^{+}$ILC2 subsets, both subsets were FACS-

584

585

586

587

588

589

590

591

592

593

594

595

596

597

598

599

600

601

602

603

604

605

606

607 sorted and cultured in vitro for 7 days in complete RPMI supplemented with $10 \%$ FBS. At the end of the culture, cells were harvested and $1.10^{5}$ cells were transferred i.v. in Rag2 ${ }^{-/-} \gamma_{c}{ }^{-/-}$mice anesthetized with isoflurane, one day before i.n. Mtb infection. For ILC1-like transfer, $1 \times 10^{4}$ purified ILC1-like were directly transferred via intratracheal (i.t.) route in mice anesthetized with isoflurane one day before Mtb infection in Rag2-/$\gamma_{c}^{-/-}$.

\section{Lung harvest}

Mice were sacrificed any cervical dislocation under isoflurane anesthesia and lungs were harvested aseptically, homogenized using a gentleMACS dissociator (C Tubes, Miltenyi) in HBSS (Difco), and incubated with DNAse I $(0.1 \mathrm{mg} / \mathrm{mL}$, Roche) and collagenase D (2 mg/mL, Roche) during $30 \mathrm{~min}$ at $37^{\circ} \mathrm{C}$ under $5 \% \mathrm{CO} 2$. When

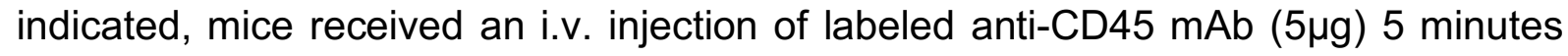
before sacrifice to discriminate between parenchymal and intravascular cells in subsequent flow cytometry analyses. Lung homogenates were filtered on $40 \mu \mathrm{m}$ cell strainers and centrifuged at $329 \times \mathrm{g}$ during $5 \mathrm{~min}$. Supernatants were conserved for cytokine content analysis. A part of the cellular pellet was conserved in TRIzol reagent for cellular RNA analysis. Bacterial loads (colony forming units) were determined by plating serial dilutions of the lung homogenates onto $7 \mathrm{H} 10$ solid medium (Difco) supplemented with $10 \%$ oleic acid-albumin-dextrose-catalase (OADC, Difco). The plates were incubated at $37^{\circ} \mathrm{C}$ for 3 weeks before bacterial CFUs scoring. In the remaining fraction, red blood cells were lysed in $150 \mathrm{mM} \mathrm{NH} 4 \mathrm{Cl}, 10 \mathrm{mM} \mathrm{KHCO}_{3}, 0.1$ mM EDTA ( $\mathrm{pH}$ 7.2) for immunological staining. 
608

609

610

611

612

613

614

615

616

617

618

619

620

621

622

623

624

625

626

627

628

629

630

631

632

633

634

635

636

637

638

639

640 Live/Dead fixable blue (eBiosciences) and mouse FcBlock (BD Biosciences) were 641 used for all flow cytometry experiments. Cell staining was analyzed using LSR

\section{In situ expansion of ILC}

To expand ILC2, C57BL/6 or Rag2 ${ }^{-/-}$mice were treated intranasally (i.n.) with $100 \mathrm{ng}$ of recombinant IL-33 (Biolegend) each day for 5 consecutive days. For the cytokinebased plasticity model, $\mathrm{C} 57 \mathrm{BL} / 6$ or Rag2 $^{-/-}$mice were treated i.n. with different combinations of cytokines specified in figures legends at day 1, 3, 5, 8 and sacrificed at day 9: $100 \mathrm{ng}$ of IL-12 (R\&D), IL-18 (R\&D), IL-33 (Biolegend) or $20 \mu \mathrm{gg}$ of NMU (US Biological) per mouse and per instillation. For the Seahorse assays we elicited ILC2 with $0.5 \mathrm{mg}$ IL-33, three doses i.p. over 10 days. Sorted ILC2 from lung were then cultured in presence of IL-7 and IL-2 $(50 \mathrm{ng} / \mathrm{ml})$ for 7 days before addition of DMOG.

\section{Flow cytometry}

To identify mouse ILCs, single-cell suspensions were stained with mAb for known lineages and with $\mathrm{mAb}$ discriminating ILC subsets. mAbs for known lineages included CD3 (17A2, Biolegend), CD4 (RM4-5, Biolegend), CD8a (53-6.7, Biolegend), TCRaß (H57-597, Biolegend), TCRyס, (GL3, Biolegend) CD11b (M1/70, Biolegend), CD11C (N418, Biolegend), F4/80 (BM8, Biolegend), Ly6G (1A8, Biolegend), TER119 (TER119, Biolegend), FceRla (MAR-1, Biolegend), CD19 (1D3/CD19, Biolegend), B220 (RA3-6B2, Biolegend), and CD49b (DX5, Biolegend). mAbs discriminating ILC subsets included CD45.2 (104, BD), CD90.2 (30-H12, Biolegend), CD127 (A7R34, eBioscience), NK1.1 (PK136, BD Biosciences), IL-18Ra (P3TUNYA, eBioscience), ST2 (RMST2-2, eBioscience), CD226 (10E5, Biolegend), and CD49a (Ha31/8), NKp46 (29A1.4). mAbs for intracellular staining included GATA3 (L50-823, BD Biosciences), T-bet (4B10, eBiosciences), ROR $\gamma \mathrm{t}$ (Q31-378, BD Biosciences), TCF-1 (S33-966, BD), Arg1 (A1exF5, BD Biosciences), Ki-67 (SolA15, eBiosciences), Eomes (Dan11mag, eBiosciences, and HIF1 $\alpha$ (D1S7W, Cell Signaling). After extracellular staining, cells were fixed and permeabilized (Foxp3 staining kit, eBiosciences) for intracellular staining. Samples from Biosafety Level 3 were inactivated for 2 hours at RT with 4\% paraformaldehyde (ThermoFisher Scientific) after extracellular and intracellular staining. 
642 Fortessa flow cytometers (BD) and FlowJo software (v10). Cells were first gated in 643 singlets (FSC-H vs. FSC-W and SSC-H vs. SSC-W) and live cells before further 644 analyses.

645

\section{Intracellular cytokines staining}

647

648 For intracellular cytokines staining of ILCs, single-cell suspensions from lung were 649 incubated at $37^{\circ} \mathrm{C}$ with Brefeldin $A$ in association or not with PMA $(50 \mathrm{ng} / \mathrm{ml}$, 650 Sigma)/lonomycine (500 ng/ml, Sigma) or $50 \mathrm{ng} / \mathrm{ml}$ of IL-12 and IL-18 for 4 hours 651 before being surface stained, fixed and permeabilized (Foxp3 staining kit, eBiosciences). mAbs for cytokines staining included IFN- $\gamma$ (XMG1.2, Biolegend), IL17A (TC11-18H10, BD Biosciences), IL-5 (TRFK5, BD Biosciences), and IL-13 (eBio13A, eBiosciences) For HIF1 $\alpha$ staining of ILCs, single-cell suspensions from lung were incubated at $37^{\circ} \mathrm{C}$ with DMOG $(500 \mu \mathrm{M})$ for 3 hours before being surface stained, fixed and permeabilized (Foxp3 staining kit, eBiosciences). In order to block glycolysis during ex vivo stimulation, cells were incubated in the presence of $10 \mathrm{mM} 2-\mathrm{DG}$ (Sigma). Mtb was inactivated by incubation in PFA 4\% for 2 hours at room temperature.

659

660

\section{ILC enrichment and cell-sorting}

661

Lung ILCs were enriched from lung single-cell suspensions by using the EasySepтм Mouse ILC2 Enrichment Kit (StemCell). After enrichment, cells were stained with

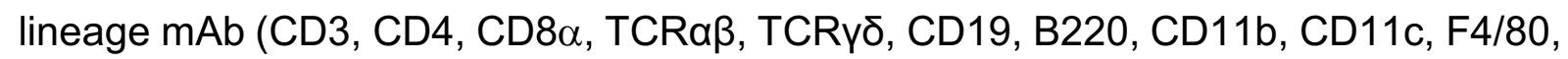
TER119, FceRla, CD49b, Ly6G) and ILC markers (CD90.2, CD45.2, NK1.1, ST2, IL18Ra, CD49a). ILC2 were purified as Lin-CD45.2 ${ }^{+}$CD90.2 ${ }^{+} \mathrm{NK} 1.1-\mathrm{ST} 2^{+}$IL-18R $\alpha^{+/-}$ILC1like were purified as Lin-CD45.2+CD90.2 ${ }^{+} \mathrm{NK} 1.1-\mathrm{ST} 2-\mathrm{CD} 49 \mathrm{a}^{+} \mathrm{IL}-18 \mathrm{Ra}^{+}$. Cells were sorted using a FACSAria Fusion cytometer (BD, France).

669

\section{In vitro culture of ILC2}

671

672 Cell sorted ILC2 were incubated in 6-well plates at a density of 300,000 cells per ml 673 for 4 days with IL-2 (25 ng/ml, R\&D) and IL-7 (25 ng/ml, R\&D) in RPMI (Difco) 674 supplemented with $10 \%$ FBS. After 4 days of culture, ILC2 were harvested for adoptive 
675 transfer or incubated with DMOG (Sigma). For DMOG experiment, half of the medium 676 was removed and replaced with fresh medium containing IL-2 (25 ng/ml) and IL-7 (25 $677 \mathrm{ng} / \mathrm{ml})$ with or without DMOG $(500 \mu \mathrm{M})$ for 3 more days. Cell sorted ILC2 were 678 incubated in 6-well plates at a density of 300,000 cells per $\mathrm{ml}$ for 4 days with IL-2 (25 $679 \mathrm{ng} / \mathrm{ml}, \mathrm{R} \& \mathrm{D})$ and IL-7 (25 ng/ml, R\&D) in RPMI (Difco) supplemented with 10 \% FBS. 680 After 4 days of culture, ILC2 were harvested for adoptive transfer or incubated with 681 DMOG (Sigma). For DMOG experiment, half of the medium was removed and 682 replaced with fresh medium containing IL-2 $(25 \mathrm{ng} / \mathrm{ml})$ and IL-7 $(25 \mathrm{ng} / \mathrm{ml})$ with or 683 without DMOG $(500 \mu \mathrm{g} / \mathrm{ml})$ for 3 more days. For the Seahorse assays, sorted ILC2 684 from lung were cultured in presence of IL-7 and IL-2 $(50 \mathrm{ng} / \mathrm{ml})$ for 7 days before addition of DMOG.

686

\section{SCENITH assay}

688

SCENITH experiments were performed as previously described (Argüello et al., 2020) using the SCENITH kit containing all reagents and anti-puromycin antibodies (requested from www.scenith.com/try-it). Briefly, lung cell suspensions were stimulated for 15 minutes at $37^{\circ} \mathrm{C}$ in the presence of the indicated inhibitors of various metabolic pathways then incubated for 30 minutes with puromycin at $37^{\circ} \mathrm{C}$. At the end of the incubation, puromycin was stained with fluorescent anti-puromycin antibodies (Clone R4743L-E8) by flow cytometry and the impact of the various metabolic inhibitors was quantitated as described (Argüello et al., 2020).

\section{Seahorse experiments}

699

1.5 to $2 \times 10^{5}$ FACS sorted lung ILC2s per well were rested in a 96-well plate in Glutamax RPMI (supplemented with 10\% fetal bovine serum, non-essential amino acids, $1 \mathrm{mM}$ sodium pyruvate, $85 \mu \mathrm{M}$ 2-mercapto-ethanol and $100 \mathrm{U} / \mathrm{ml}$ penicillinstreptomycin) containing $25 \mathrm{ng} / \mathrm{ml} \mathrm{IL-7.} \mathrm{After} 24 \mathrm{~h}$ cells were split and rested in fresh IL-7 containing media for another 3 days. Subsequently, cells were cultured in fresh medium containing $25 \mathrm{ng} / \mathrm{ml} \mathrm{IL-7}$ and $20 \mathrm{ng} / \mathrm{ml}$ of IL-2 in the presence or absence of $0.5 \mathrm{mM}$ DMOG for a further 72 hours. To prepare for extracellular flux analysis cells were then washed thoroughly in XF medium (modified DMEM) and adhered to the 
709 For glycolytic stress test, cells were plated at a density of $2 \times 10^{5}$ cells/well in XF medium 710 supplemented with $2 \mathrm{mM}$ glutamine. Cells were incubated for $30-60 \mathrm{~min}$ at $37^{\circ} \mathrm{C}$ and 711 ECAR was measured under basal conditions, and in response to $10 \mathrm{mM}$ glucose, 2 $712 \mu \mathrm{M}$ oligomycin and $50 \mathrm{mM}$ 2-DG. For the mitochondrial stress test, cells were plated 713 at a density of $1.5 \times 10^{5}$ cells/well in XF medium supplemented with $2 \mathrm{mM}$ glutamine, 1 $714 \mathrm{mM}$ sodium pyruvate and $25 \mathrm{mM}$ glucose. Cells were incubated for $30-60 \mathrm{~min}$ at $37^{\circ} \mathrm{C}$. 715 OCAR was measured under basal conditions, after injection of $2 \mu \mathrm{M}$ oligomycin, 1.5 $\mu \mathrm{M}$ FCCP and $100 \mathrm{nM}$ rotenone $+1 \mu \mathrm{M}$ antimycin A. Extracellular flux assays were done using a 96-well extracellular flux analyzer XFe-96 (Seahorse Bioscience).

Normalization by protein was used to correct for potential differences in seeding densities across wells. Protein measurement was performed using the Pierce BCA protein assay according to the manufacturer instructions.

Quantitative RT-PCR analysis of transcripts

RNA from lungs homogenates was extracted using TRIzol reagent (Ambion) and RNeasy spin columns according to manufacturer's instructions (RNeasy kit, Qiagen). RNA was reverse transcribed into cDNA using M-MLV Reverse transcriptase (Invitrogen). RT-qPCR was performed using gene-targeted primers (Supplementary Table 1) as described above. Values were normalized using the housekeeping betaactin gene (Actb) and expressed as a fold change. RNA from ILC2 culture were extracted using RLT (Qiagen) and RNA were reverse transcribed as previously described (Troegeler et al., 2017).

\section{Statistical analyses}

Statistical analyses were performed using GraphPad Prism 9 software. Agostino and Pearson normality tests were performed to determine whether data followed a normal distribution. Unpaired $t$-test (for normal data) or Mann-Whitney (for non-normal data) were performed when two samples were compared; ANOVA (for normal data) or Kruskal-Wallis (for non-normal data) tests were performed when more than two samples were compared. For all analyses, * indicates $\mathrm{P}<0.05$, ${ }^{* *}$ indicates $\mathrm{P}<0.01$, *** indicates $P<0.001$, and ${ }^{* * * *}$ indicates $P<0.0001$. 


\section{Acknowledgements}

744 We thank Yasmine Belkaid (NIH/NIAID, Bethesda) for critical review of the manuscript. We acknowledge Emmanuelle Näser (Genotoul TRI-IPBS platform, Toulouse) for flow cytometry and cell-sorting, Flavie Moreau, Céline Berrone and Aline Tridon (Genotoul Anexplo-IPBS platform, Toulouse), and Sylvie Appolinaire and Celine Berraud (CREFRE US006, Toulouse) for mouse care and maintenance in conventional and BSL3 facilities. We thank Richard Locksley (USCF, San Francisco) for the kind gift of Red5 mice. We thank Geanncarlo Lugo-Villarino, Sophie Laffont (CPTP, Toulouse) and Andrea Pichler (CRCT, Toulouse) for helpful discussions. This work was supported by Centre de la Recherche Scientifique (CNRS), the University Toulouse III-Paul Sabatier, the French Ministry for Higher Education, Research and Innovation (fellowship to D.C.), the Fondation pour la Recherche Médicale (grant DEQ20160334902 to O.N.), the Bettencourt Schueller Foundation (grants Coup d'Élan pour la recherche française and Explore-TB to O.N.), MSDAVENIR (grant Fight-TB to O.N.), the Agence Nationale de la Recherche (grants ANR-18-CE15-0004-01 to D.H. and ANR-11-EQUIPEX-0003 to O.N.), and the European Commission (grant TBVAC2020 n643381 to O.N.). M.R.H. is supported by a Royal Society and Wellcome Trust Sir Henry Dale fellowship (105644/Z/14/Z), a Lister Institute of Preventative Medicine Prize and a BBSRC Project grant (BB/T014482/1). The funders had no role in study design, data collection, and analysis, decision to publish, or preparation of the manuscript.

\section{Author contributions}

D.C. and D.H. conceived and designed the study with input from O.N.; D.C., A.C., M.Z.K., E.B., T.S. and F.L. performed the experiments; E.L., J-P.G., and R.J.A contributed critical reagents and methods, D.C., G.E., J-C.G., R.J.A., M.R.H. and D.H. analyzed and interpreted the data; J-C.G., G.E., M.R.H., and Y.P., also provide important discussion for the project and critical feedback on the manuscript; D.C., O.N. and D.H. wrote the manuscript. All coauthors read, reviewed and approved the manuscript. 
bioRxiv preprint doi: https://doi.org/10.1101/2021.01.19.427257; this version posted July 13, 2021. The copyright holder for this preprint (which was not certified by peer review) is the author/funder. All rights reserved. No reuse allowed without permission.

776

777 The authors declare no competing interests 


\section{Figure legends}

Figure 1. IL-18R $\alpha$-expressing ILC2 differentiate into ILC1-like cells during Mtb

781

782

783

784

785

786

787

788

789

790

791

792

793

794

795

796

797

798

799

800

801

802

803

804

805

806

807

808

809

infection (A) Representative dot plots showing the gating strategy used to analyze ILC subsets in the lungs of C57BL/6 mice after doublets exclusion (top graphs): ILC1 (dark blue), ILC3 (red), IL-18R $\alpha^{-}$ILC2 (green), IL-18R $\alpha^{+}$ILC2 (yellow) and T-bet ${ }^{+}$IL$18 \mathrm{R} \alpha^{+}$ILC (light blue) are depicted in non-infected and Mtb-infected mice. (B) Unsupervised t-SNE distribution of total lung Lin-CD90.2+ $2^{+}$populations at steady-state (left graph) and during Mtb infection (right graph). Based on gating strategy defined in Figure 1A, ILC subsets were depicted with the same color code: ILC1 in dark blue, ILC3 in red, IL-18R $\alpha^{-}$ILC2 in green, IL-18R $\alpha^{+}$ILC2 in yellow and ILC1-like cells (light blue). (C) Expression of GATA3 (MFI), ST2 (\%), and Arg1 (\%) in indicated ILC subsets at day 28 post-infection in C57BL/6 mice. (D) Expression of T-bet (MFI), CD49a (\%) and CD226 (MFI) in indicated ILC subsets at day 28 post-infection in C57BL/6 mice. (E) Percentages of IFN- $\gamma$, IL-5, and IL-17A positive cells in the indicated ILC subsets after ex vivo stimulation with PMA/ionomycin in presence of Brefeldin A for $4 \mathrm{~h}$ at day 28 post-infection in C57BL/6 mice. (F) Absolute numbers of ILC1-like cells at the indicated days after Mtb infection. Prior to sacrifice, mice were injected with fluorescent anti-CD45.2 to distinguish vascular and parenchymal cells. ILC1-like cells have been gated on lung-resident cells. (G) Percentages of IFN- $\gamma^{+}$cells in the indicated ILC subsets after ex vivo stimulation with IL-12+IL-18 or not, in presence of Brefeldin A for 4h at day 28 post-infection in C57BL/6 mice. $(\mathrm{H})$ Absolute numbers of IL-18R $\alpha^{+}$ILC2 at the indicated days after Mtb infection. Prior to sacrifice, mice were injected with fluorescent anti-CD45.2 to distinguish vascular and parenchymal cells. IL-18R $\alpha^{+}$ILC2 have been gated on lung-resident cells. (I) Experimental settings for the adoptive transfer of IL-18R $\alpha^{-}$and IL-18R $\alpha^{+}$ILC2 into Rag2 $2^{-/-} \gamma \mathrm{C}^{-/-}$before Mtb infection. (J) Percentage of ILC (Lin-CD45. $2^{+}$CD $90.2^{+}$CD $\left.127^{+}\right)$in lung at day 21 post-infection in $\mathrm{Rag}^{-{ }^{-}} \gamma \mathrm{C}^{-/}$after adoptive transfer of IL-18R $\alpha^{-}$ILC2 (green) and IL-18R $\alpha^{+}$ILC2 (yellow). (K) As in (J), but for T-bet expression in ILC. In (C, D, F and H), data are representative of five independent experiments, in (E, G) data are representative of two independent experiments and in $(\mathbf{J}, \mathbf{K})$ data are a pool of two independent experiments with each symbol representing an individual mouse, graphs depict data as mean ( \pm s.e.m) and 
810 statistical analysis was performed using two-way (E-F), one-way ANOVA (C-D, G-H)

811 or Mann Whitney test $(\mathbf{J}-\mathrm{K})\left({ }^{*}, \mathrm{p}<0.05 ;{ }^{* *}, \mathrm{P}<0.01 ;{ }^{* * *}, \mathrm{p}<0.001 ;{ }^{* * *}, \mathrm{p}<0.0001\right)$. 
812

813

814

815

816

817

818

819

820

821

822

823

824

825

826

827

828

829

830

831

832

833

834

835

836

837

838

839

840

\section{9}

Figure 2. The inflammatory environment shapes the fate of IL-18R $\alpha^{+}$ILC2. (A) Representative dot-plots of T-bet and IL18R $\alpha$ expression after intranasal administration of PBS and IL-12+IL-18 in Rag2-/- mice in Lin-CD45.2 ${ }^{+}$CD90.2 ${ }^{+} \mathrm{NK} 1.1^{-}$ ROR $\gamma t^{-}$cells (B) Absolute numbers of IL-18R $\alpha^{-}$ILC2 (green), IL-18R $\alpha^{+}$ILC2 (yellow) and ILC1-like cells (blue) after cytokine (IL-12+IL-18) or control (PBS) treatment. (C) Percentage of TCF-1 in lung IL-18R $\alpha^{-}$ILC2 after intranasal administration of PBS (white dots) or IL-12+IL-18 (grey dots). (D) Percentages of cells expressing IL-5 or IFN$\gamma$ among the indicated ILC subsets after ex vivo stimulation with PMA/ionomycin in the presence of brefeldin A for $4 \mathrm{~h}$. (E) Percentages of IFN- $\gamma^{+}$cells in the indicated ILC subsets after ex vivo stimulation with IL-12+IL-18, PMA/ionomycin or not in presence of Brefeldin A for $4 \mathrm{~h}$ from IL-12+IL-18 treated C57BL/6 mice. (F) Representative dotplots of T-bet and IL18Ra expression after intranasal administration of IL-33, IL-12+IL18 and IL-12+IL-18+IL-33 in Rag2-/- mice in Lin-CD45.2 ${ }^{+}$CD90.2+NK1.1-ROR ${ }^{+}{ }^{-}$cells. (G-I) Absolute numbers of IL-18R $\alpha^{-}$ILC2 (G), IL-18R $\alpha^{+}$ILC2 (H) and ILC1-like cells (I) after intranasal administration of IL-33, IL-12+IL-18, or IL-12+IL-18+IL-33. (J) Expression of ST2 (\%), Arg1 (\%) and CD49a (\%) in IL-18R $\alpha^{+}$ILC2 in IL-33 (black dots), IL-12+IL-18 (white dots) and IL-12+IL-18+IL-33 (grey dots) -treated mice. (K) Percentage of IL-5 IFN- $\gamma^{-}$(right), IL-5-IFN- $\gamma^{+}$(middle), IL-5+IFN- $\gamma^{+}$(left) in IL-18R $\alpha^{+}$ ILC2 in IL-33 (black dots), IL-12+IL-18 (white dots) and IL-12+IL-18+IL-33 (grey dots) -treated mice. (L) Absolute numbers of IL-18R $\alpha^{+}$ILC2 (left) and ILC1-like cells (right) after intranasal administration of PBS (control), neuromedin $U$ (NMU), IL-12+IL-18, or IL-12+IL-18+NMU. Each symbol represents an individual mouse. Statistical analysis was performed using Mann-Whitney (B, C), one-way (G-K) and two-way (D-E, L) ANOVA tests $\left({ }^{*}, \mathrm{p}<0.05 ;{ }^{* *}, \mathrm{P}<0.01 ;{ }^{* * *}, \mathrm{p}<0.001 ;{ }^{* * *}, \mathrm{p}<0.0001\right)$. Graphs depict data as mean $( \pm$ s.e.m). Data are representative of three $(\mathbf{B}, \mathbf{D}, \mathbf{G}-\mathbf{K})$, and two $(\mathbf{C}, \mathbf{E}, \mathbf{L})$ independent experiments. 
841 Figure 3. Metabolic reprogramming toward glycolysis is associated with an ILC1-

842 like cell differentiation. (A) Representative histograms of puromycin staining in NK cells (violet), IL-18R $\alpha^{-}$ILC2 (green), IL-18R $\alpha^{+}$ILC2 (yellow), and ILC1-like cells (blue)

844

845

846

847

848

849

850

851

852

853

854

855

856

857

858

859

860

861

862

863

864

865

866

867

868

869

870

871

872 in IL-12+IL-18-treated mice. (B) Expression of puromycin (MFI) in NK cells (violet), IL18R $\alpha^{-}$ILC2 (green), IL-18R $\alpha^{+}$ILC2 (yellow), and ILC1-like cells (blue) in PBS vs. IL12+IL-18 treated mice. (C) Percentages of puromycin-positive cells in NK cells (violet), IL-18R $\alpha^{-}$ILC2 (green), IL-18R $\alpha^{+}$ILC2 (yellow), and ILC1-like cells (blue) in PBS vs. IL-12+IL-18 treated mice. (D) Representative histograms of puromycin staining (left) and quantification (MFI, right) in ILC1-like cells from IL-12+IL-18 treated mice after incubation with various metabolic inhibitors (Co, control; DG, 2-Deoxyglycose; O, oligomycin; DGO, 2-Deoxyglucose + Oligomycin). (E-F) Percentages of mitochondrial dependence (E), and glycolytic capacity (F) in NK (violet), IL-18R $\alpha^{-}$ILC2 (green), IL18R $\alpha^{+}$ILC2 (yellow), and ILC1-like cells (blue) in PBS vs. IL-12+IL-18 treated mice. (G) Percentages of Arg1+ cells among IL-18R $\alpha^{-}$ILC2 (green), IL-18R $\alpha^{+}$ILC2 (yellow), and ILC1-like cells (blue) defined after intranasal administration of PBS (control) or cytokines (IL-12+IL-18). (H) Histograms showing HIF1 $\alpha$ expression in IL-18R $\alpha^{-}$ILC2 (left), IL-18R $\alpha^{+}$ILC2 (middle) and ILC1-like cells in PBS (black line) or IL-12+IL-18 (blue line) treated mice. Dash line represents isotype control for HIF1 $\alpha$ staining. Due to the absence of ILC1-like cells at steady-state, only the condition with IL-12+IL-18 treatment is represented. (I) Histograms showing HIF1 $\alpha$ protein expression in ILC2 cultured in the absence (vehicle, grey) or presence of DMOG (blue). The dot line represents FMO for HIF1 $\alpha$ detection. (J-L) Quantitative analysis of the intensity of GATA3 (J), ST2 (K) and IL-5 (L) expression in ILC2 cultured in the absence (vehicle) or presence of DMOG. (M) Seahorse analysis of glycolytic stress test with quantification of glycolysis and glycolytic capacity of ILC2 cultured in the presence or absence of DMOG. (N) Seahorse analysis of mitochondrial respiration with quantification of spare respiratory capacity of ILC2 cultured in the presence or absence of DMOG. (O) as in (J-L) except that the expression of Tbx21, Ifng and I/18ra1 mRNA was analyzed by RT-qPCR. Each symbol represents an individual mouse and statistical analysis was performed using two-way ANOVA (B, C, E, F, G), and paired $t$ test $(\mathbf{J}-\mathbf{O})\left({ }^{*}, \mathrm{p}<0.05 ;{ }^{* *}, \mathrm{P}<0.01 ;{ }^{* * *}, \mathrm{p}<0.001 ;{ }^{* * *}, \mathrm{p}<0.0001\right)$. Graphs depict data as mean $( \pm$ s.e.m) from two $(\mathbf{A}-\mathbf{N})$ and a pool of three $(\mathbf{O})$ independent experiments. 
873 Figure 4. ILC1-like cells confer protection against Mtb. (A) C57BL/6 were

874 vaccinated by internasal administration of BCG or not (PBS) 60 days before infection.

875 After 14 days post-infection, mice were euthanized. (B) Mycobacterial loads at day 14 876 post-infection in C57BL/6 mice vaccinated or not (PBS) with $1 \times 10^{5}$ BCG via the 877 intranasal route 60 days prior Mtb infection. (C) Percentages of total lung ILC 878 expressing T-bet. (D) Absolute numbers of ILC1 (dark blue), ILC3 (red), IL-18R $\alpha^{-}$ILC2 879 (green), IL-18R $\alpha^{+}$ILC2 (yellow) and ILC1-like cells (light blue) at day 14 post-infection 880 in C57BL/6 mice vaccinated or not (PBS) with $1 \times 10^{5} \mathrm{BCG}$ via the intranasal route 60 881 days prior Mtb infection. (E) Percentages of IFN- $\gamma^{+}$cells among ILC1-like cells in Mtb882 infected unvaccinated vs. vaccinated mice after ex vivo stimulation with IL-12+IL-18 in 883 the presence of brefeldin A for $4 \mathrm{~h}$. (F) Schematic representation of the in vivo 884 expansion of ILC1-like cells in Rag2-/- mice treated with IL-12+IL-18+IL-33, cell-sorting 885 of ILC1-like cells (Lin-CD45.2 ${ }^{+}$CD90.2 $2^{+}$NK1.1-ST2-CD49a ${ }^{+}$IL-18R $\alpha^{+}$) and adoptive 886 transfer in $\mathrm{Rag}^{-/-} / / 2 \mathrm{rg}^{-/-}$one day before infection with $\mathrm{Mtb}$ by intratracheal route. (G) 887 Representative histograms of T-bet, GATA3 and ROR $\gamma$ t expression in sorted ILC1-like 888 cells (grey) vs. ILC2 (Lin-CD45.2 ${ }^{+}$CD90.2 ${ }^{+}$NK1.1-ST2 ${ }^{+}$cells). (H) Bacterial loads at day 88921 post-infection in Rag2 $^{-/-I} / 2 \mathrm{rg}^{-/-}$mice having received (+ILC1-like) or not (-ILC1like) 890 an adoptive transfer of ILC1-like cells from IL-12+IL-18+IL-33 treated Rag2-/- mice one 891 day before Mtb infection. Each symbol represents an individual mouse. Statistical 892 analysis was performed using Mann-Whitney test $(\mathrm{B}-\mathrm{H})\left({ }^{*}, \mathrm{p}<0.05 ;{ }^{* *}, \mathrm{P}<0.01 ;{ }^{* * *}\right.$, $\left.893 \mathrm{p}<0.001 ;{ }^{* * * *}, \mathrm{p}<0.0001\right)$. Graphs depict data as mean ( \pm s.e.m). Data are 894 representative of two (B-H) independent experiments. 
895 Supplementary Figure 1. Dynamics of ILCs during Mtb infection in the mouse 896 model. (A) Representative expression of GATA3 and ST2 on the indicated ILC 897 subsets. (B) Expression of GATA3 (MFI), ST2 (\%) and Arg1 (\%) in ILC1 (blue), IL898 $18 \mathrm{R} \alpha^{-}$ILC2 (green) and IL-18R $\alpha^{+}$ILC2 (yellow) at steady-state in the lung of C57BL/6 mice. (C) Expression of IL-5 (\%) and IFN- $\gamma(\%)$ in the indicated ILC subsets after ex 900 vivo stimulation with PMA/ionomycin in presence of Brefeldin $A$ for $4 \mathrm{~h}$ at steady-state 901 in the lung of C57BL/6 mice (D) Percentages of TCF-1+ ${ }^{+}$cells in ILC2P from bone marrow (grey) compared to IL-18R $\alpha^{-}$ILC2 (green) and IL-18R $\alpha^{+}$ILC2 (yellow) from 903 the lungs of C57BL/6 mice at steady-state. (E) Percentages of $\mathrm{NKp} 46^{+}$(left) and Eomes $^{+}$(right) cells in ILC1-like cells compared to in NK cells and ILC1 at day 28 post905 infection. (F) Absolute numbers of ILC1 (dark blue), ILC3 (red) and IL-18R $\alpha^{-}$ILC2 at 906 the indicated days after Mtb infection. Prior to sacrifice, mice were injected with fluorescent anti-CD45.2 to distinguish vascular and parenchymal cells. ILC1, ILC3, IL$18 \mathrm{R} \alpha^{-}$ILC2 and IL-18R $\alpha^{+}$ILC2 have been gated on lung-resident cells. (G) Absolute number of ILC1-like cells at day 28 post-infection in C57BL/5 (grey) vs. Rag2-/- (black) mice. (H) Schematic representation of the in vivo expansion of ILC2 in C57BL/6 or Rag2 ${ }^{-/-}$mice treated with IL-33, cell-sorting, in vitro culture and adoptive transfer of ILC2 in Rag2 $2^{-/} / / 2 \mathrm{rg}^{-/-}$one day before infection with Mtb. (I) Gating strategy to purify ILC2 based on the expression of ST2 (left two graphs) and purity of ILC2 after cellsorting (right). (J) Phenotype of ILC2 after in vitro culture and before adoptive transfer.

915 (K) A representative dot-plot of GATA3 and T-bet expression in Lin-CD45.2 ${ }^{+}$CD90.2 ${ }^{+}$ 916 cells isolated from $\mathrm{Rag}^{-/-} / / 2 \mathrm{rg}^{-/-}$mice adoptively transferred with purified ILC2 then left uninfected (right) or infected with Mtb (left). (L) Percentages of T-bet expressing ILC at different days post-infection. (M) Expression of IL-18R $\alpha$ (\%) and $\mathrm{Ki67}(\%)$ in 919 transferred GATA3 ${ }^{+}$(white dots) vs. T-bet ${ }^{+}$(grey dots) ILC at day 28 post-infection in

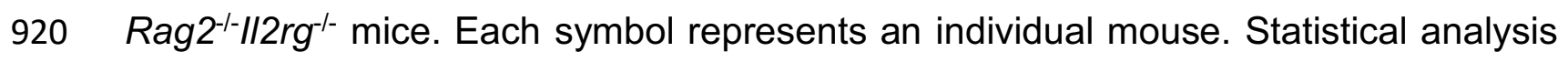
921 was performed using one-way ANOVA test (B-F, L) or Mann-Whitney test (G, M) (* $\left.922 \mathrm{p}<0.05 ;{ }^{* *}, \mathrm{P}<0.01 ;{ }^{* * *}, \mathrm{p}<0.001 ;{ }^{* * *}, \mathrm{p}<0.0001\right)$. Graphs depict data as mean $( \pm$ 923 s.e.m). Data are representative of five (F), three (B-C, K-M) and two (D, E, G) 924 independent experiments. 


\section{Supplementary Figure 2. Combination of IL-12, IL-18 and IL-33 induces a mixed}

926 ILC1 and ILC2 phenotype on IL-18R $\alpha^{+}$ILC2. (A) Representative dot-plot showing IL-

9275 and IL-18R $\alpha$ expression among IL-18R $\alpha^{-}$ILC2 (green), IL-18R $\alpha^{+}$ILC2 (red), and 928 ILC1-like (blue) in PBS vs. IL-12+IL-18 treated IL-5 ${ }^{\text {Cre ROSA26 YFP }}$ mic (left) and 929 quantification (right). (B) Representative dot-plot showing YFP and IL-18R $\alpha$ 930 expression among IL-18R $\alpha^{-}$ILC2 (green), IL-18R $\alpha^{+}$ILC2 (red), and ILC1-like (blue) in 931 PBS vs. IL-12+IL-18 treated IL-5 ${ }^{\text {CreROSA26 }}{ }^{\text {YFP }}$ mic (left) and quantification (right). (C) 932 Unsupervised t-SNE representation of the expression of different markers (GATA3, 933 Arg1, T-bet, IL-18R $\alpha$, CD49a, CD226 and Ki67) on Lin-CD45.2 ${ }^{+}$CD90.2 ${ }^{+} \mathrm{NK} 1.1^{-}{ }^{-}$OR $\gamma t^{-}$ 934 in non-infected vs. Mtb-infected (28 dpi) vs. IL-12+IL-18 treated Rag2-/- mice. (D) As in 935 A) except that the analysis was performed on Lin-CD45.2 ${ }^{+}$CD $90.2^{+} \mathrm{NK} 1.1^{-} \mathrm{ROR} \gamma \mathrm{t}^{-}$in IL$93612+\mathrm{IL}-18$ vs. IL-33 vs. IL-12+IL-18 treated Rag2 ${ }^{-/-}$mice. Statistical analysis was 937 performed two-way ANOVA test (A-B) $\left(^{*}, \mathrm{p}<0.05 ;{ }^{* *}, \mathrm{P}<0.01 ;{ }^{* * *}, \mathrm{p}<0.001\right.$; ${ }^{* * *}$, 938 p<0.0001). Graphs depict data as mean ( \pm s.e.m). Data are representative of two (AB) independent experiments. 
940 Supplementary Figure 3. Glycolysis regulates ILC1-like cell differentiation

941 during Mtb infection. (A) Percentage of puromycin positive cells in ILC1 (dark blue), 942 ILC3 (red), IL-18R $\alpha^{-}$ILC2 (green), IL-18R $\alpha^{+}$ILC2 (yellow) and ILC1-like cells (blue) in 943 non-infected vs. Mtb-infected Rag2 ${ }^{-/-}$mice. (B) HIF1 $\alpha$ expression in the indicated ILC 944 subsets at day 28 post-infection (C) Expression of IFN- $\gamma$ in total ILCs after ex vivo 945 stimulation with IL-12+IL-18 in the presence or absence of 2-DG. (D) Absolute 946 numbers of ILC1-like cells in Rag2 ${ }^{-/-}$mice treated or not with 2-DG during Mtb infection 947 at day 28 post-infection. (E) Percentages of IFNy-producing cells among ILC1-like cells 948 after ex vivo stimulation with PMA/ionomycin in the presence of brefeldin A for $4 \mathrm{~h}$ from 949 PBS vs. 2-DG treated mice. (F) As in (D) except that mice treated with $30 \%$ glucose in 950 their drinking water. (G) As in (E) except that mice were treated or not with $30 \%$ glucose 951 in their drinking water. Each symbol represents an individual mouse and statistical 952 analysis was performed using two-way ANOVA (A), one-way ANOVA (B), Wilcoxon 953 (C) and Mann-Whitney (D-G) tests $\left({ }^{*}, p<0.05 ;{ }^{* *}, P<0.01 ;{ }^{* * *}, p<0.001 ;{ }^{* * *}, p<0.0001\right)$. 954 Graphs depict data as mean ( \pm s.e.m) from three (D-E) or two (A-C, F-G) independent 955 experiments. 
956 Supplementary Figure 4. Metabolic regulation of IL-18R $\alpha^{+}$ILC2 differentiation 957 into ILC1-like cells during Mycobacterium tuberculosis infection. Mtb infection 958 results in the establishment of a type 1 inflammation. Type 1 cytokines, IL-12, IL-18 959 and IFN- $\gamma$ (upper part) act on a rare, lung immature IL-18R $\alpha$-expressing ILC2 subset 960 and triggers a glycolysis-involving metabolic reprogramming leading to its 961 differentiation into ILC1-like cells IL18R $\alpha^{+}$, CD49a ${ }^{+}$CD226 ${ }^{+} H I F-1 \alpha^{+}$, T-bet $^{+}$, IFN- $\gamma-$ 962 producing) endowed with a protective potential against Mtb. In contrast, type 2 963 cytokines such as IL-33 (lower part) acts on this immature IL-18R $\alpha$-expressing ILC2 964 subset to drive its maturation toward mature IL-5 producing ILC2. 
A

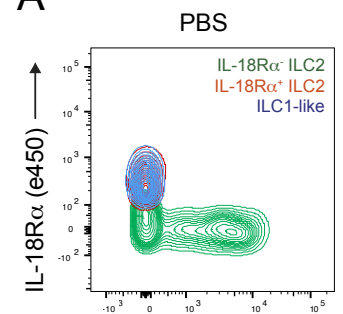

Red5 (IL-5) $\longrightarrow$

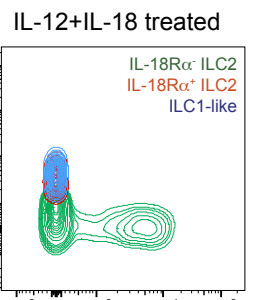

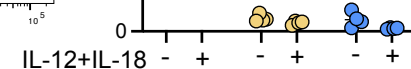

B

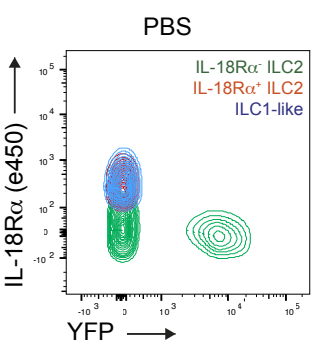

D
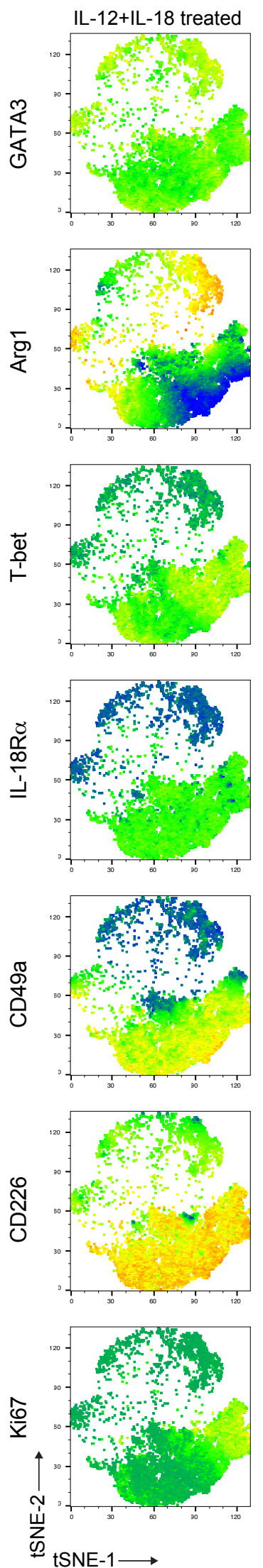

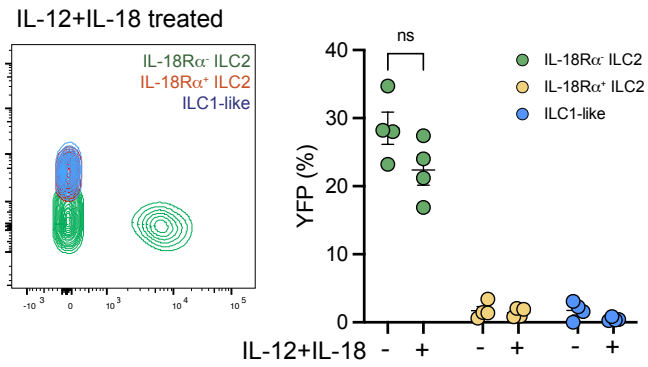

IL-12+IL-18 -

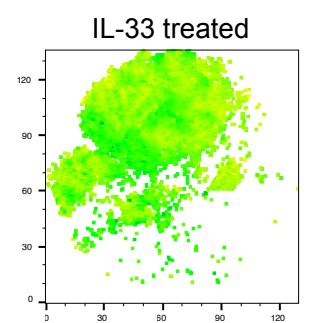

IL-12+IL-18+IL-33 treated
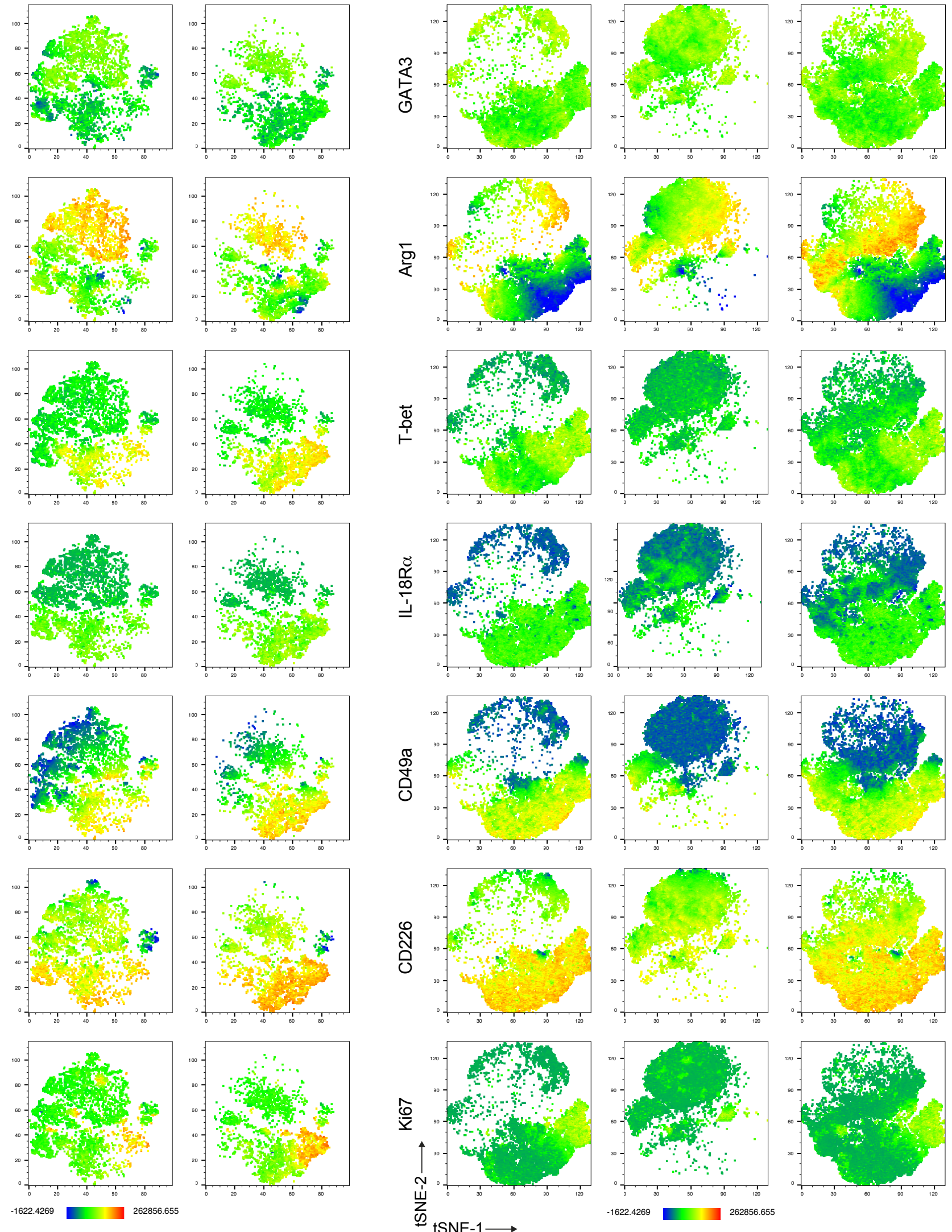
A

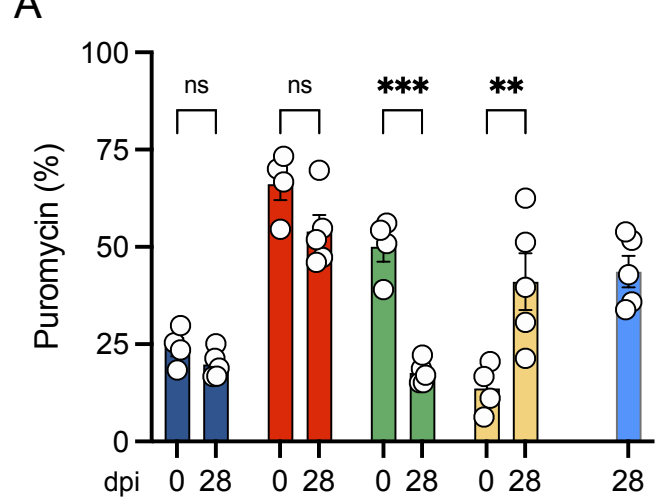

B

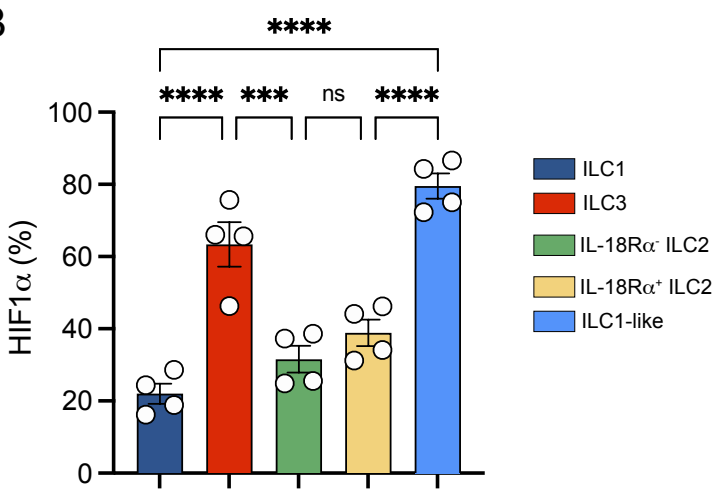

C

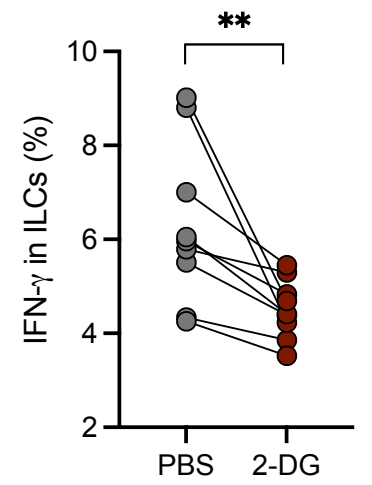

D

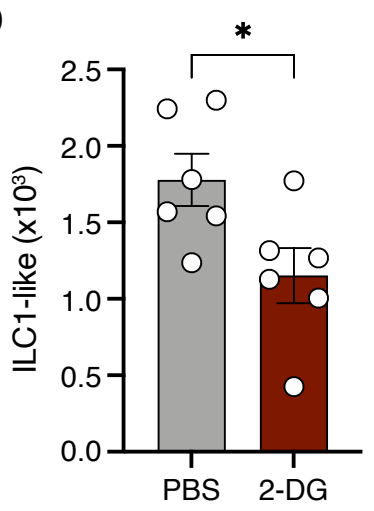

E

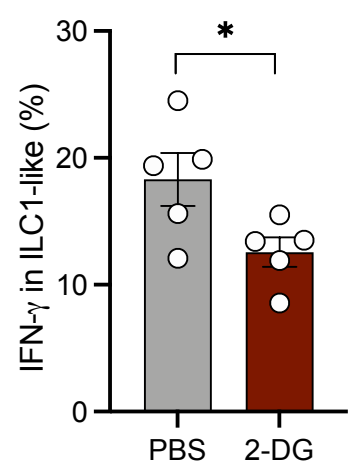

F

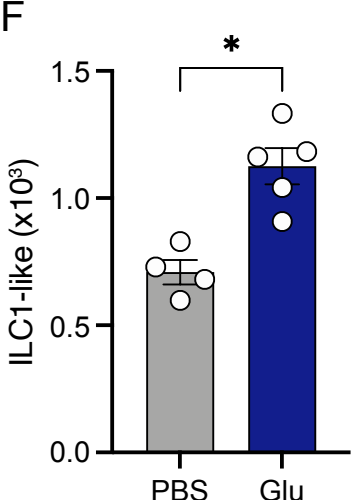

G

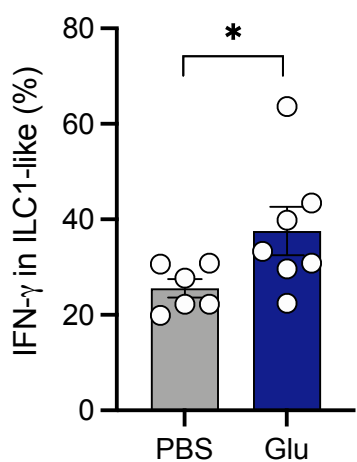


Corral et adioRFiviqupentAdoi: https://doi.org/10.1101/2021.01.19.427257; this version posted July 13, 2021. The copyright holder for this preprint (which was not certified by peer review) is the author/funder. All rights reserved. No reuse allowed without permission.

A

Intranasal administration of BCG or PBS (control)

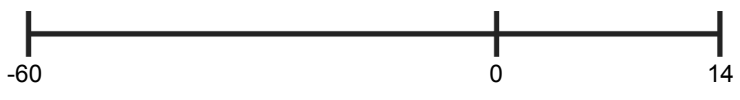

$\mathrm{B}$

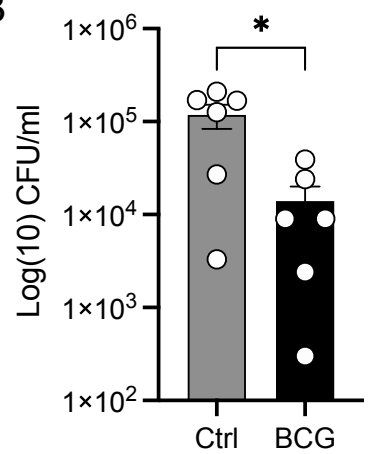

C

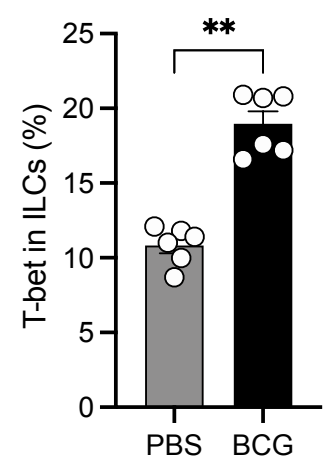

D

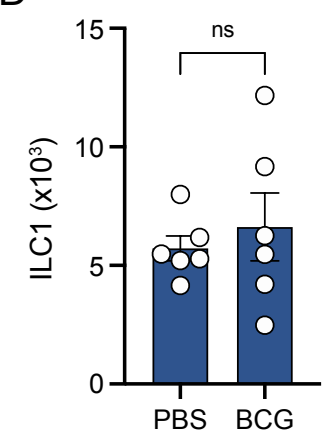

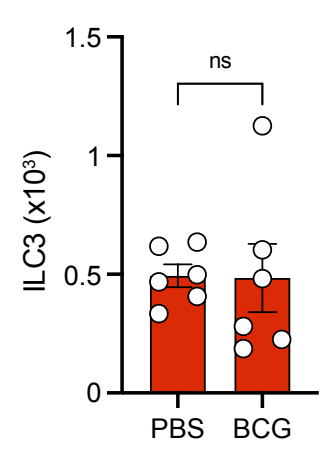

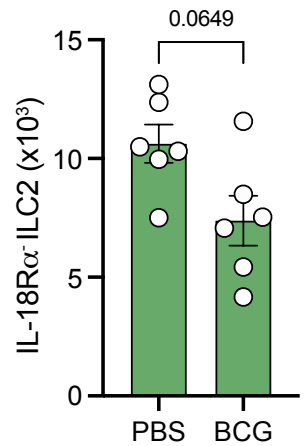

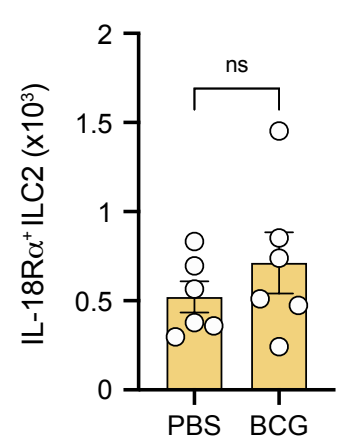
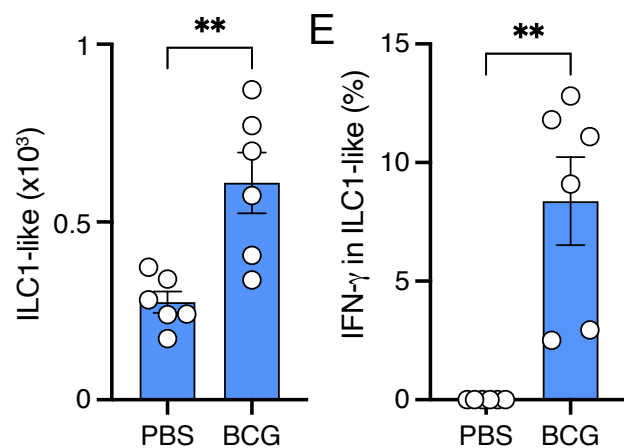

$\mathrm{F}$

G

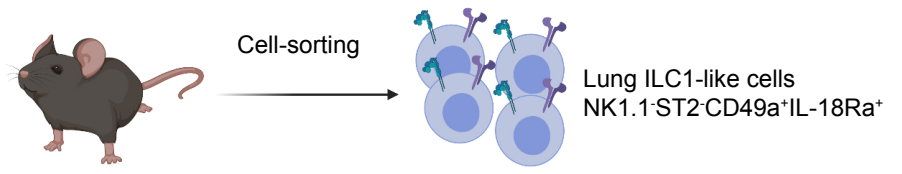

Intratracheal injection of $1.10^{4}$ ILC1-like cells

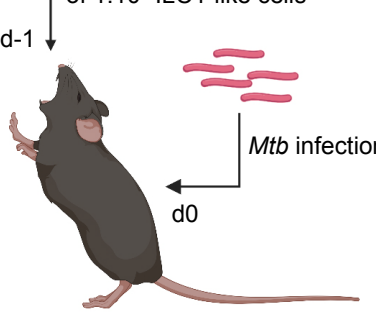

Rag2 $2^{-1 /} \mathrm{C}^{-/-}$

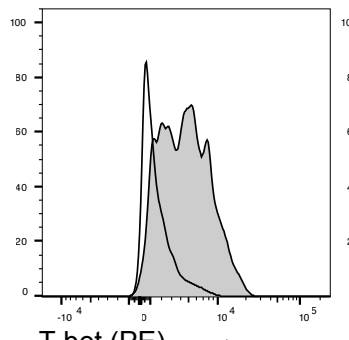
T-bet (PE)
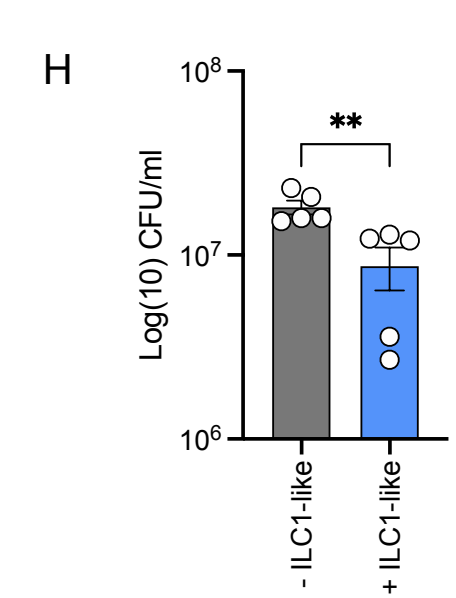


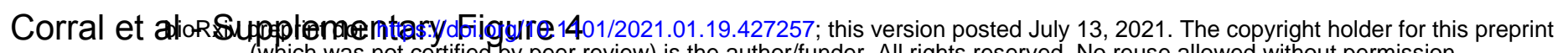
(which was not certifiedby peer review) is the author/funder. All rights reserved. No reuse allowed without permission.

Mycobacterium tuberculosis
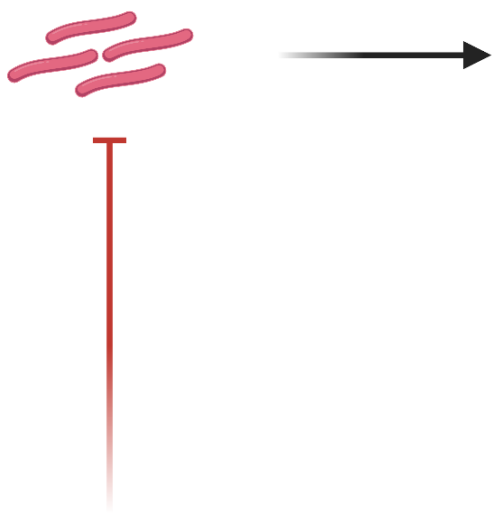

Type 1 Inflammation

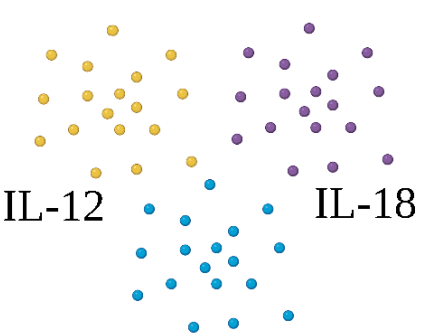

IFN- $\gamma$

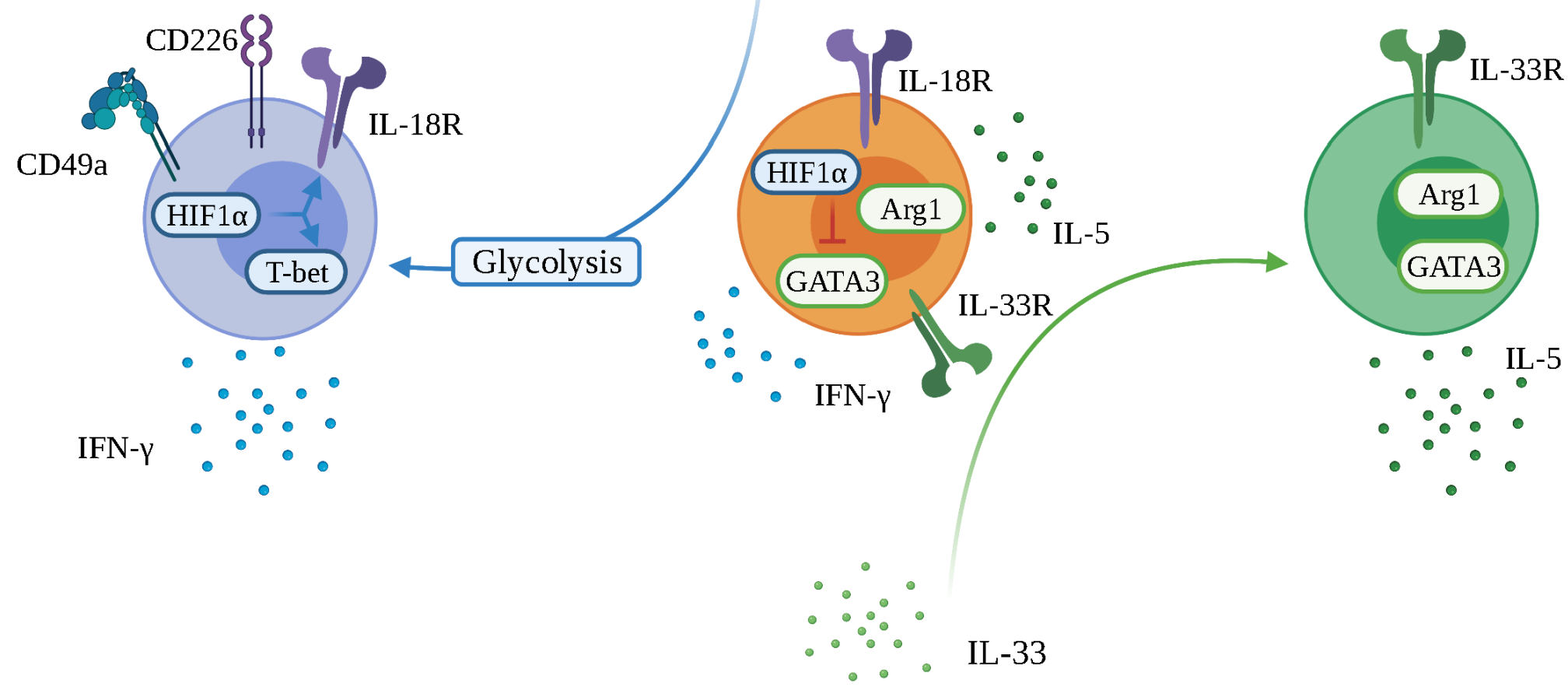

Type 2 Inflammation

Created with BioRender.com 